CERN-TH-99-151

ECM-UB-PF-99-12

hep-ph/9906210

\title{
Effective Transport Equations for non-Abelian Plasmas
}

\author{
Daniel F. Litim f $^{a}$ and Cristina Manuel fib \\ ${ }^{a}$ Departament ECM \& IFAE, Facultat de Física \\ Univ. de Barcelona, Diagonal 647, E-08028 Barcelona, Spain. \\ ${ }^{b}$ Theory Division, CERN, CH-1211 Geneva 23, Switzerland.
}

\begin{abstract}
Starting from classical transport theory, we derive a set of covariant equations describing the dynamics of mean fields and their statistical fluctuations in a nonAbelian plasma in or out of equilibrium. A general procedure is detailed for integrating-out the fluctuations as to obtain the effective transport equations for the mean fields. In this manner, collision integrals for Boltzmann equations are obtained as correlators of fluctuations. The formalism is applied to a hot non-Abelian plasma close to equilibrium. We integrate-out explicitly the fluctuations with typical momenta of the Debye mass, and obtain the collision integral in a leading logarithmic approximation. We also identify a source for stochastic noise. The resulting dynamical equations are of the Boltzmann-Langevin type. While our approach is based on classical physics, we also give the necessary generalizations to study the quantum plasmas. Ultimately, the dynamical equations for soft and ultra-soft fields change only in the value for the Debye mass.
\end{abstract}

\footnotetext{
*E-Mail: Litim@ecm.ub.es

${ }^{\dagger}$ E-Mail: Cristina.Manuel@cern.ch
} 


\section{INTRODUCTION}

This article presents in full detail an approach to the dynamics of non-Abelian plasmas based on classical transport theory, the main results of which have been summarized in [ []].

In the recent years, there has been an increasing interest in the dynamics of non-Abelian plasmas at very high temperatures or high densities. Due to the asymptotic freedom of quantum chromodynamics, one expects that quarks and gluons are no longer confined under such extreme conditions, but rather behave as free entities forming the so-called quark-gluon plasma. Within the next few years, a lot of efforts will be given to detect experimentally this new state of matter using heavy-ion colliders. Another domain of application concerns the physics of the early universe. If baryogenesis can finally be understood within an electroweak scenario, an understanding of the physics of the electroweak model in the high temperature regime where the spontaneous broken symmetry is restored, is essential for a computation of the rate of baryon number violation.

It is therefore mandatory to devise reliable theoretical tools for a quantitative description of non-Abelian plasmas both in or out of equilibrium. While some progress has been achieved in the recent years [2], we are still far away from having a satisfactory understanding of the relevant relaxation and transport processes in non-Abelian plasmas, in particular when it comes to out-of-equilibrium situations.

There are different approaches in the literature to study non-Abelian plasmas, ranging from thermal field theory to quantum transport equations or lattice studies. Even in the close-to-equilibrium plasma, and for small gauge coupling, the situation is complicated due to the non-perturbative character of long-wavelength excitations in the plasma. Most attempts to tackle this problem are based on a quantum field theoretical description of the non-Abelian interactions [ [4 4 . It has been conjectured that the plasma close to equilibrium allows for a description in terms of soft classical fields, as the occupation number for the soft excitations are large. While a classical transport theory approach for the non-Abelian case [5] is known to reproduce the one-loop thermal effective action [6], it has never been exploited in full detail. The opposite holds true for Coulomb plasmas, where all the essential transport phenomena have been studied longly using techniques developed within (semi-)classical kinetic theory [7, while a quantum field theoretical approach has been undertaken only recently.

Our approach aims at filling this gap in the literature of classical non-Abelian plasmas. Here, we follow the philosophy of Klimontovitch [7], and our equations can be seen as the generalization of classical kinetic theory for Abelian plasmas to non-Abelian ones. Our essential contribution is considering the non-Abelian colour charges as dynamical variables and introducing the concept of ensemble average to the non-Abelian kinetic equations. Equally important is the consistent treatment of the intrinsic non-linearities of non-Abelian gauge interactions. The observation that Klimontovitch's procedure leads to the BalescuLenard collision integral for Coulomb plasmas has motivated earlier derivations of similar (semi-)classical kinetic equations for non-Abelian plasmas [8 [10]. However, these implementations are not fully consistent, and have never been worked out in all generality.

The starting point for a classical transport theory of non-Abelian plasmas is considering an ensemble of classical point particles carrying a non-Abelian charge. They interact 
through self-consistent fields, that is, the fields generated by the particles themselves. The microscopic dynamics is governed by the classical equations of motion given by the Wong equations [1]. When the number of particles is large, one has to abandon a microscopic description of the system in favour of a macroscopical one based on an ensemble average of all the microscopic quantities. This leads naturally to a description in terms of averaged quantities, and their statistical fluctuations. By averaging the microscopic dynamical equations, we obtain effective transport equations for mean quantities. These contain the collision integrals of the macroscopic Boltzmann equation, which appear in this formulation as statistical correlators of fluctuating quantities. By subtracting the exact microscopic equations from the mean ones, we obtain the dynamical equations for the fluctuations themselves. In principle, these two set of equations should be enough to consider all the transport phenomena in the plasma.

This method is then applied in full detail to a thermal non-Abelian plasma close to equilibrium, which allows to employ some approximations. For a small plasma parameter, the two-particle correlators are small and can be neglected. In the case of small fluctuations, the dynamical equations simplify considerably. These conditions are always met for a small gauge coupling parameter, which, for simplicity, will be assumed throughout. Our approximate equations are the leading order ones in a consistent expansion in the gauge coupling. However, we shall also see that the condition for a kinetic description to be valid could also be met for large gauge couplings. After taking statistical averages, we are able to explicitly integrate-out the fluctuations with momenta about the Debye mass. This gives the collision integrals which appear in the transport equations for the mean fields. In addition to the dissipative processes in the plasma described by the collision term, we are able to deduce the stochastic source which prevents the system from abandoning equilibrium. This is an important result, because it allows to prove explicitly that the fluctuation-dissipation theorem holds, when switching from a microscopic to a macroscopic description of the system. These findings for classical plasmas can be generalized to the case of quantum ones. The resulting dynamical equation match perfectly the effective theory for the ultra-soft modes as found by other approaches 12 14.

The lesson to be learned is thus two-fold: There exists a fully self-contained formalism to study classical non-Abelian plasmas in the first place, which opens in particular a door for applications to out-of-equilibrium situations. Second, this approach is -technically speakingmuch easier than approaches based on the full quantum field theory. Some of the intrinsic complications of a quantum field theoretical description (like gauge-fixing, ghost degrees of freedom) can be avoided, and in the close to equilibrium plasma, the same effective dynamical equations are obtained.

The paper is organized as follows. We begin with a review of the microscopic picture, based on the classical equations of motions for coloured point particles (sect. II). Changing to a macroscopic description needs the introduction of a statistical average, which also allows the computation of correlators of fluctuations (sect. III). This procedure is then applied to the fields and the distribution function as to obtain dynamical equations for their mean values and their fluctuations. The dynamical equations are given in its most general form. Possible approximation schemes are detailed, and the interpretation of statistical correlators in terms of collision integrals is given (sect. [V]). The consistency of the procedure 
with the requirements of gauge invariance is shown for the complete set of equations, and some approximations to them (sect. V). In order to apply the formalism to a plasma close to equilibrium, we discuss first the relevant physical scales for both classical and quantum plasmas (sect. V1). This is followed by a fully detailed derivation of the mean field dynamical equation for classical plasmas, which includes the integrating-out of the fluctuations with momenta about the Debye mass, and the computation of the collision integral and the related noise variable in a leading logarithmic approximation. A brief discussion of (iterative) solutions to the Boltzmann-Langevin equation for different momentum regimes is also presented (sect. VII). We argue that these results can be translated to the case of quantum plasmas and detail the necessary changes. Some comments on related work are added as well (sect. VIII). Finally, we present our conclusions (sect. [X]), deferring to the appendices some technical details regarding the Darboux variables for $\mathrm{SU}(N)$ colour charges (appendix A), and the derivation of a useful algebraic identity (appendix B).

\section{MICROSCOPIC EQUATIONS FOR NON-ABELIAN CHARGED PARTICLES}

Let us consider a system of particles carrying a colour charge $Q^{a}$, where the colour index runs from $a=1$ to $N^{2}-1$ for a $\mathrm{SU}(N)$ gauge group. Within a microscopic description, the trajectories in phase space are known exactly. The trajectories $\hat{x}(\tau), \hat{p}(\tau)$ and $\hat{Q}(\tau)$ for every particle are solutions of their classical equations of motions, the Wong equations [11

$$
\begin{aligned}
& m \frac{d \hat{x}^{\mu}}{d \tau}=\hat{p}^{\mu}, \\
& m \frac{d \hat{p}^{\mu}}{d \tau}=g \hat{Q}^{a} F_{a}^{\mu \nu} \hat{p}_{\nu}, \\
& m \frac{d \hat{Q}^{a}}{d \tau}=-g f^{a b c} \hat{p}^{\mu} A_{\mu}^{b} \hat{Q}^{c} .
\end{aligned}
$$

Here, $A_{\mu}$ denotes the microscopic gauge field. The corresponding microscopic field strength $F_{\mu \nu}^{a}$ and the energy momentum tensor of the gauge fields $\Theta^{\mu \nu}$ are given by

$$
\begin{aligned}
F_{\mu \nu}^{a}[A] & =\partial_{\mu} A_{\mu}^{a}-\partial_{\nu} A_{\mu}^{a}+g f^{a b c} A_{\mu}^{b} A_{\nu}^{c}, \\
\Theta^{\mu \nu}[A] & =\frac{1}{4} g^{\mu \nu} F_{\rho \sigma}^{a} F_{a}^{\rho \sigma}+F_{a}^{\mu \rho} F_{\rho}^{a \nu}
\end{aligned}
$$

and $f^{a b c}$ are the structure constants of $\mathrm{SU}(N)$. We set $c=k_{B}=\hbar=1$ and work in natural units, unless otherwise indicated. Note that the non-Abelian charges are also subject to dynamical evolution. Equation (2.1G) can be rewritten as $D_{\tau} Q=0$, where $D_{\tau}=\frac{d \hat{x}^{\mu}}{d \tau} D_{\mu}$ is the covariant derivative along the world line, and $D_{\mu}^{a c}[A]=\partial_{\mu} \delta^{a c}+g f^{a b c} A_{\mu}^{b}$ the covariant derivative in the adjoint representation. With $Q_{a}$ and $F_{\mu \nu}^{a}$ transforming in the adjoint representation, the Wong equations can be shown to be invariant under gauge transformations. The equation (2.10) ensures the conservation under dynamical evolution of the set of $N-1$ Casimir of the $S U(N)$ group.

\footnotetext{
${ }^{\ddagger}$ For $S U(2)$, it is easy to verify explicitly the conservation of the quadratic Casimir $Q_{a} Q_{a}$. For $S U(3)$, both the quadratic and cubic Casimir $d_{a b c} Q_{a} Q_{b} Q_{c}$, where $d_{a b c}$ are the symmetric structure
} 
The colour current associated to each particle can be constructed once the solutions of the Wong equations are known. For every single particle it reads

$$
j_{a}^{\mu}(x)=g \int d \tau \frac{d \hat{x}^{\mu}}{d \tau} \hat{Q}_{a}(\tau) \delta^{(4)}(x-\hat{x}(\tau))
$$

Employing the Wong equations (2.1) we find that $j^{\mu}$ is covariantly conserved, $D_{\mu} j^{\mu}=0$ [11]. Similarly, the energy momentum tensor associated to a single particle is given by [11]

$$
t^{\mu \nu}(x)=\int d \tau \frac{d \hat{x}^{\mu}}{d \tau} \hat{p}^{\nu}(\tau) \delta^{(4)}(x-\hat{x}(\tau))
$$

It is convenient to describe the ensemble of particles introducing a phase space density which depends on the whole set of coordinates $x^{\mu}, p^{\mu}$ and $Q_{a}$. We define the function

$$
n(x, p, Q)=\sum_{i} \int d \tau \delta^{(4)}\left(x-\hat{x}_{i}(\tau)\right) \delta^{(4)}\left(p-\hat{p}_{i}(\tau)\right) \delta^{\left(N^{2}-1\right)}\left(Q-\hat{Q}_{i}(\tau)\right)
$$

where the index $i$ labels the particles. This distribution function is constructed in such a way that the colour current

$$
J_{a}^{\mu}(x)=g \int d^{4} p d^{\left(N^{2}-1\right)} Q \frac{p^{\mu}}{m} Q_{a} n(x, p, Q)
$$

coincides with the sum over all currents associated to the individual particles, $J_{a}^{\mu}=\sum_{i} j_{a}^{\mu}$, and is covariantly conserved, $D_{\mu} J^{\mu}=0$. It is convenient to make the following changes in the choice of the distribution function. We will define a new function $f(x, p, Q)$ such that the physical constraints like the on-mass shell condition, positive energy and conservation of the group Casimirs are factored out into the phase space measure. We introduce the momentum measure

$$
d P=d^{4} p 2 \theta\left(p_{0}\right) \delta\left(p^{2}-m^{2}\right)
$$

and the measure for the colour charges

$$
d Q=d^{3} Q c_{R} \delta\left(Q_{a} Q_{a}-q_{2}\right)
$$

in the case of $\mathrm{SU}(2)$. For $S U(3)$ the measure is

$$
d Q=d^{8} Q c_{R} \delta\left(Q_{a} Q_{a}-q_{2}\right) \delta\left(d_{a b c} Q^{a} Q^{b} Q^{c}-q_{3}\right) .
$$

constants of the group, are conserved under the dynamical evolution. The last conservation can be checked using (2.10) and a Jacobi-like identity which involves the symmetric $d_{a b c}$ and antisymmetric $f_{a b c}$ constants.

$\S$ Note that in [1] has an additional factor of $(2 \pi)^{3}$. 
For $S U(N), N-1 \delta$-functions ensuring the conservation of the set of $N-1$ Casimirs have to be introduced into the measure $d Q$. We have also introduced the representation-dependent normalization constant $c_{R}$ into the measure $d Q$, which is fixed by requiring $\int d Q=1$ (see appendix A]. The constant $C_{2}$ is defined as

$$
\int d Q Q_{a} Q_{b}=C_{2} \delta_{a b}
$$

and depends on the group representation of the particles.

With these conventions the colour current (2.7) reads now

$$
J_{a}^{\mu}(x)=g \int d P d Q p^{\mu} Q_{a} f(x, p, Q)
$$

while the energy momentum tensor associated to the particles obtains as

$$
t^{\mu \nu}(x)=\int d P d Q p^{\mu} p^{\nu} f(x, p, Q) .
$$

We will now come to the dynamical equation of the microscopic distribution functions $n(x, p, Q)$ and $f(x, p, Q)$, which will serve as the starting point for the subsequent formalism. The dynamical equation for $n(x, p, Q)$ is the same as for $f(x, p, Q)$. This is so because the physical constraints which we have factored out as to obtain $f(x, p, Q)$ are not affected by the Wong equations. Employing (2.1), we find

$$
p^{\mu}\left(\frac{\partial}{\partial x^{\mu}}-g f^{a b c} A_{\mu}^{b} Q^{c} \frac{\partial}{\partial Q^{a}}-g Q_{a} F_{\mu \nu}^{a} \frac{\partial}{\partial p_{\nu}}\right) f(x, p, Q)=0,
$$

which can be checked explicitly by direct inspection of (2.6) into 2.14a (see appendix A of [6]). In a self-consistent picture this equation is completed with the Yang-Mills equation,

$$
\left(D_{\mu} F^{\mu \nu}\right)_{a}(x)=J_{a}^{\nu}(x)
$$

and the current being given by (2.12). It is worth noticing that (2.14) is exact in the sense that no approximations have been made so far. The effects of collisions are included inasmuch as the Wong equations do account for them, although (2.14a) looks formally like a collisionless Boltzmann equation.

For the energy momentum tensor of the gauge fields we find

$$
\partial_{\mu} \Theta^{\mu \nu}=-F_{a}^{\nu \mu} J_{\mu}^{a}
$$

On the other hand, using (2.14a) and the definition (2.13) we find that

$$
\partial_{\mu} t^{\mu \nu}=F_{a}^{\nu \mu} J_{\mu}^{a}
$$

which establishes that the total energy momentum tensor is conserved, $\partial_{\mu}\left(\Theta^{\mu \nu}+t^{\mu \nu}\right)=0$.

To finish the review of the microscopic description of the system, let us recall the gauge symmetry properties of the set of equations (2.14) (see [6] for more details). From the 
definition of $f(x, p, Q)$ it follows that it transforms as a scalar under a (finite) gauge transformation, $f^{\prime}\left(x, p, Q^{\prime}\right)=f(x, p, Q)$. This implies the gauge covariance of $(2.14 \mathrm{~b})$ because the current (2.7) transforms like the vector $Q_{a}$ in the adjoint. The non-trivial dependence of $f$ on the non-Abelian colour charges implies that the partial derivative $\partial_{\mu} f$ does not yet transform as a scalar, but rather its covariant derivative $D_{\mu} f$, which is given by

$$
D_{\mu}[A] f(x, p, Q) \equiv\left[\partial_{\mu}-g f^{a b c} Q_{c} A_{\mu, b} \partial_{a}^{Q}\right] f(x, p, Q) .
$$

Notice that (2.17) combines the first two terms of (2.14a). (Here, and in the sequel we use the shorthand notation $\partial_{\mu} \equiv \partial / \partial x^{\mu}, \partial_{\mu}^{p} \equiv \partial / \partial p^{\mu}$ and $\partial_{a}^{Q} \equiv \partial / \partial Q^{a}$.) The invariance of the third term in (2.14a) follows from the trivial observation that $Q_{a} F_{\mu \nu}^{a}$ is invariant under gauge transformations. This establishes the gauge invariance of (2.14a).

\section{STATISTICAL AVERAGES}

If the system under study contains a large number of particles it is impossible to follow their individual trajectories. One has then to switch to a statistical description of the system.

In this section, we describe in detail the statistical average to be used in the sequel. As we are studying classical point particles in phase space, the appropriate statistical average corresponds to the Gibbs ensemble average for classical systems. We will review the main features of this procedure, defined in phase space. Let us remark that this derivation is completely general, valid for any classical system, and does not require equilibrium situations.

We will introduce two basic functions. The first one is the phase space density function $\mathcal{N}$ which gives, after integration over a phase space volume element, the number of particles contained in that volume. In a microscopic description it is just a deterministic quantity, and a function of the time $t$, the vectors $\mathbf{x}$ and $\mathbf{p}$, and the set of canonical (Darboux) variables $\boldsymbol{\phi}$ and $\boldsymbol{\pi}$ associated to the colour charges $Q$ (see appendix A). For $S U(N)$, there are $N(N-1) / 2$ pairs of canonical variables, which we denote as $\phi=\left(\phi_{1}, \ldots, \phi_{N(N-1) / 2}\right)$ and $\boldsymbol{\pi}=\left(\pi_{1}, \ldots, \pi_{N(N-1) / 2}\right)$. The microscopic phase space density is given by

$$
\mathcal{N}(\mathbf{x}, \mathbf{p}, \boldsymbol{\phi}, \boldsymbol{\pi})=\sum_{i} \delta^{(3)}\left(\mathbf{x}-\hat{\mathbf{x}}_{i}(t)\right) \delta^{(3)}\left(\mathbf{p}-\hat{\mathbf{p}}_{i}(t)\right) \delta\left(\boldsymbol{\phi}-\hat{\boldsymbol{\phi}}_{i}(t)\right) \delta\left(\boldsymbol{\pi}-\hat{\boldsymbol{\pi}}_{i}(t)\right),
$$

where the sum runs over all particles of the system, and $\left(\hat{\mathbf{x}}_{i}, \hat{\mathbf{p}}_{i}, \hat{\boldsymbol{\phi}}_{i}, \hat{\boldsymbol{\pi}}_{i}\right)$ refers to the trajectory of the $i$-th particle in phase space. Then $\mathcal{N} d \mathbf{x} d \mathbf{p} d \boldsymbol{\phi} d \boldsymbol{\pi}$ gives the number of particles at time $t$ in an infinitesimal volume element of phase space around the point $z=(\mathbf{x}, \mathbf{p}, \boldsymbol{\phi}, \boldsymbol{\pi})$

Let us now define the distribution function $\mathcal{F}$ of the microstates of a system of $L$ identical classical particles. Due to Liouville's theorem, $d \mathcal{F} / d t=0$. Thus, it can be normalized as

$$
\int d z_{1} d z_{2} \ldots d z_{L} \mathcal{F}\left(z_{1}, z_{2}, \ldots, z_{L}, t\right)=1 .
$$

Here $z_{i}$ denotes all the phase space variables associated to the particle $i$. For simplicity we have considered that there is only one species of particles in the system, although the generalization to several species of particles is rather straightforward. 
The statistical average of any function $\mathcal{G}$ defined in phase space is given by

$$
\langle\mathcal{G}\rangle=\int d z_{1} d z_{2} \ldots d z_{L} \mathcal{G}\left(z_{1}, z_{2}, \ldots, z_{L}\right) \mathcal{F}\left(z_{1}, z_{2}, \ldots, z_{L}, t\right)
$$

The one-particle distribution function is obtained from $\mathcal{F}$ as

$$
f_{1}\left(z_{1}, t\right)=V \int d z_{2} \ldots d z_{L} \mathcal{F}\left(z_{1}, z_{2}, \ldots, z_{L}, t\right)
$$

where $V$ denotes the phase space volume. Correspondingly, the two-particle distribution function is

$$
f_{2}\left(z_{1}, z_{2}, t\right)=V^{2} \int d z_{3} \ldots d z_{L} \mathcal{F}\left(z_{1}, z_{2}, \ldots, z_{L}, t\right)
$$

and similarly for the $k$-particle distribution functions. A complete knowledge of $\mathcal{F}$ would allow us to obtain all the set of $\left(f_{1}, f_{2}, \ldots, f_{L}\right)$ functions; this is, however, not necessary for our present purposes.

Notice that we have allowed for an explicit dependence on the time $t$ of the function $\mathcal{F}$, as this would be typically the case in out of equilibrium situations. We will drop this $t$ dependence from now on to simplify the formulas.

Using the above definition one can obtain the mean value of the microscopic phase space density. Microscopically $\mathcal{N}(z)=\sum_{i=1}^{L} \delta\left(z-\hat{z}_{i}\right)$, where $\hat{z}_{i}$ describes the trajectory in phase space of the particle $i$. The statistical average of this function is

$$
\langle\mathcal{N}(z)\rangle=\int d z_{1} d z_{2} \ldots d z_{L} \mathcal{F}\left(z_{1}, z_{2}, \ldots, z_{L}\right) \sum_{i=1}^{L} \delta\left(z-\hat{z}_{i}\right)=\frac{L}{V} f_{1}(z) .
$$

The second moment $\left\langle\mathcal{N}(z) \mathcal{N}\left(z^{\prime}\right)\right\rangle$ can equally be computed, and it is not difficult to see that gives

$$
\left\langle\mathcal{N}(z) \mathcal{N}\left(z^{\prime}\right)\right\rangle=\frac{L}{V} \delta\left(z-z^{\prime}\right) f_{1}(z)+\frac{L(L-1)}{V^{2}} f_{2}\left(z, z^{\prime}\right) .
$$

Let us now define a deviation of the phase space density from its mean value

$$
\delta \mathcal{N}(z) \equiv \mathcal{N}(z)-\langle\mathcal{N}(z)\rangle
$$

By definition $\langle\delta \mathcal{N}(z)\rangle=0$, although the second moment of this statistical fluctuation does not vanish in general, since

$$
\left\langle\delta \mathcal{N}(z) \delta \mathcal{N}\left(z^{\prime}\right)\right\rangle=\left\langle\mathcal{N}(z) \mathcal{N}\left(z^{\prime}\right)\right\rangle-\langle\mathcal{N}(z)\rangle\left\langle\mathcal{N}\left(z^{\prime}\right)\right\rangle
$$

If the number of particles is large, $L \gg 1$, we have

$$
\left\langle\delta \mathcal{N}(z) \delta \mathcal{N}\left(z^{\prime}\right)\right\rangle=\left(\frac{L}{V}\right) \delta\left(z-z^{\prime}\right) f_{1}(z)+\left(\frac{L}{V}\right)^{2} g_{2}\left(z, z^{\prime}\right)
$$

where the function $g_{2}\left(z, z^{\prime}\right)=f_{2}\left(z, z^{\prime}\right)-f_{1}(z) f_{1}\left(z^{\prime}\right)$ measures the two-particle correlations in the system. For a completely uncorrelated system $g_{2}=0$. 
Notice that the above statistical averages are well defined in the thermodynamic limit, $L, V \rightarrow \infty$ but $L / V$ remaining constant. Higher order moments and higher order correlators can as well be defined.

We finally point out that the the function $\mathcal{N}(\mathbf{x}, \mathbf{p}, \boldsymbol{\phi}, \boldsymbol{\pi})$ agrees with the microscopic function $f(x, p, Q)$ introduced earlier, except for a representation-dependent normalization constant $c_{R}$ as introduced in sect. II. We will also swallow the density factors $L / V$ into the mean functions $\bar{f}$. Those small changes in the normalizations allow to simplify slightly the notations of the equations.

\section{AVERAGING THE MICROSCOPIC EQUATIONS}

\section{A. The mean fields and the fluctuations}

In this section we perform the step from a microscopic to a macroscopic formulation of the problem. Using the prescription explained in sect. III, we take statistical averages of the microscopic equations (2.14). This implies that the distribution function $f(x, p, Q)$, which in the microscopic picture is a deterministic quantity, has now a probabilistic nature and can be considered as a random function, given by its mean value and statistical (random) fluctuation about it. Let us define the quantities

$$
\begin{aligned}
f(x, p, Q) & =\bar{f}(x, p, Q)+\delta f(x, p, Q), \\
J_{a}^{\mu}(x) & =\bar{J}_{a}^{\mu}(x)+\delta J_{a}^{\mu}(x) \\
A_{\mu}^{a}(x) & =\bar{A}_{\mu}^{a}(x)+a_{\mu}^{a}(x)
\end{aligned}
$$

where the quantities carrying a bar denote the mean values, e.g. $\bar{f}=\langle f\rangle, \bar{J}=\langle J\rangle$ and $\bar{A}=\langle A\rangle$, while the mean value of the statistical fluctuations vanish by definition, $\langle\delta f\rangle=0$, $\langle\delta J\rangle=0$ and $\langle a\rangle=0$. This separation into mean fields and statistical, random fluctuations corresponds effectively to a split into low frequency (long wavelength) modes associated to the mean fields, and high frequency (short wavelength) modes associated to the fluctuations..** We also split the field strength tensor as

$$
\begin{aligned}
F_{\mu \nu}^{a} & =\bar{F}_{\mu \nu}^{a}+f_{\mu \nu}^{a}, \\
\bar{F}_{\mu \nu}^{a} & =F_{\mu \nu}^{a}[\bar{A}], \\
f_{\mu \nu}^{a} & =\left(\bar{D}_{\mu} a_{\nu}-\bar{D}_{\nu} a_{\mu}\right)^{a}+g f^{a b c} a_{\mu}^{b} a_{\nu}^{c},
\end{aligned}
$$

using also $\bar{D}_{\mu} \equiv D_{\mu}[\bar{A}]$. The term $f_{\mu \nu}^{a}$ contains terms linear and quadratic in the fluctuations. Note that the statistical average of the field strength $\left\langle F_{\mu \nu}^{a}\right\rangle$ is given by $\left\langle F_{\mu \nu}^{a}\right\rangle=\bar{F}_{\mu \nu}^{a}+$ $g f^{a b c}\left\langle a_{\mu}^{b} a_{\nu}^{c}\right\rangle$, due to quadratic terms contained in $f_{\mu \nu}^{a}$.

In the same light, we split the energy momentum tensor of the gauge fields into the part from the mean fields and the fluctuations, according to

\footnotetext{
${ }^{* *}$ In the close-to-equilibrium plasma (sect. VII), we identify the relevant momentum scales explicitly.
} 


$$
\begin{aligned}
\Theta^{\mu \nu} & =\bar{\Theta}^{\mu \nu}+\theta^{\mu \nu} \\
\bar{\Theta}^{\mu \nu} & =\frac{1}{4} g^{\mu \nu} \bar{F}_{\rho \sigma}^{a} \bar{F}_{a}^{\rho \sigma}+\bar{F}_{a}^{\mu \rho} \bar{F}_{\rho}^{a \nu}, \\
\theta^{\mu \nu} & =\frac{1}{2} g^{\mu \nu} \bar{F}_{\rho \sigma}^{a} f_{a}^{\rho \sigma}+\bar{F}_{a}^{\mu \rho} f_{\rho \nu}^{a}+\bar{F}_{a}^{\nu \rho} f_{\rho \mu}^{a}+\frac{1}{4} g^{\mu \nu} f_{\rho \sigma, a} f^{\rho \sigma, a}+f_{a}^{\mu \rho} f_{\rho}^{a \nu},
\end{aligned}
$$

The term $\theta^{\mu \nu}$ contains the fluctuations up to quartic order. Due to the non-linear character of the theory, we find that the ensemble average of the energy momentum tensor reads $\left\langle\Theta^{\mu \nu}\right\rangle=\bar{\Theta}^{\mu \nu}+\left\langle\theta^{\mu \nu}\right\rangle$.

\section{B. Dynamical equations for the mean fields and the fluctuations}

We perform now the step from the microscopic to the macroscopic Boltzmann equation, taking the statistical average of (2.14). This yields the dynamical equation for the mean values,

$$
p^{\mu}\left(\bar{D}_{\mu}-g Q_{a} \bar{F}_{\mu \nu}^{a} \partial_{p}^{\nu}\right) \bar{f}=\langle\eta\rangle+\langle\xi\rangle .
$$

We have made use of the covariant derivative of $f$ as introduced in (2.17). The macroscopic Yang-Mills equations are obtained as

$$
\bar{D}_{\mu} \bar{F}^{\mu \nu}+\left\langle J_{\text {fluc }}^{\nu}\right\rangle=\bar{J}^{\nu} .
$$

In (4.4), we collected all terms quadratic or cubic in the fluctuations into the functions $\eta(x, p, Q), \xi(x, p, Q)$ and $J_{\text {fluc }}(x)$. They read explicitly

$$
\begin{aligned}
\eta(x, p, Q) & \equiv g Q_{a} p^{\mu} \partial_{p}^{\nu} f_{\mu \nu}^{a} \delta f(x, p, Q), \\
\xi(x, p, Q) & \equiv g p^{\mu} f^{a b c} Q^{c}\left(\partial_{a}^{Q} a_{\mu}^{b} \delta f(x, p, Q)+g a_{\mu}^{a} a_{\nu}^{b} \partial_{p}^{\nu} \bar{f}(x, p, Q)\right), \\
J_{\text {fluc }}^{a, \nu}(x) & \equiv g\left\{f^{d b c} \bar{D}_{a d}^{\mu} a_{b, \mu} a_{c}^{\nu}+f^{a b c} a_{b, \mu}\left(\left(\bar{D}^{\mu} a^{\nu}-\bar{D}^{\nu} a^{\mu}\right)_{c}+g f^{c d e} a_{d}^{\mu} a_{e}^{\nu}\right)\right\}(x) .
\end{aligned}
$$

The corresponding equations for the fluctuations are obtained by subtracting (4.4) from (2.14). The result is

$$
\begin{aligned}
p^{\mu}\left(\bar{D}_{\mu}-g Q_{a} \bar{F}_{\mu \nu}^{a} \partial_{p}^{\nu}\right) \delta f= & g Q_{a}\left(\bar{D}_{\mu} a_{\nu}-\bar{D}_{\nu} a_{\mu}\right)^{a} p^{\mu} \partial_{\nu}^{p} \bar{f} \\
& +g p^{\mu} a_{b, \mu} f^{a b c} Q_{c} \partial_{a}^{Q} \bar{f}+\eta+\xi-\langle\eta+\xi\rangle \\
\left(\bar{D}^{2} a^{\mu}-\bar{D}^{\mu}\left(\bar{D}_{\nu} a^{\nu}\right)\right)^{a}+ & 2 g f^{a b c} \bar{F}_{b}^{\mu \nu} a_{c, \nu}+J_{\text {fluc }}^{a, \mu}-\left\langle J_{\text {fluc }}^{a, \mu}\right\rangle=\delta J^{a, \mu} .
\end{aligned}
$$

The above set of dynamical equations is enough to describe all transport phenomena in the plasma.

While the dynamics of the mean fields (4.4) depends on correlators quadratic and cubic in the fluctuations, the dynamical equations for the fluctuations (4.6) do in addition depend on higher order terms (up to cubic order) in the fluctuations themselves. The dynamical equations for the higher order correlation functions are contained in (4.6). To see this, consider for example the dynamical equation for the correlators $\langle\delta f \delta f\rangle$. After multiplying (4.6a) with $\delta f$ and taking the statistical average, we obtain 


$$
\begin{aligned}
p^{\mu}\left(\bar{D}_{\mu}-g Q_{a} \bar{F}_{\mu \nu}^{a} \partial_{p}^{\nu}\right)\langle\delta f \delta f\rangle= & g Q_{a} p^{\mu} \partial_{\nu}^{p} \bar{f}\left\langle\left(\bar{D}_{\mu} a_{\nu}-\bar{D}_{\nu} a_{\mu}\right)^{a} \delta f\right\rangle \\
& +g p^{\mu} f^{a b c} Q_{c} \partial_{a}^{Q} \bar{f}\left\langle a_{b, \mu} \delta f\right\rangle+\langle(\eta+\xi) \delta f\rangle .
\end{aligned}
$$

In the same way, we find for $\langle\delta f \delta f \delta f\rangle$ the dynamical equation

$$
\begin{aligned}
p^{\mu}\left(\bar{D}_{\mu}-g Q_{a} \bar{F}_{\mu \nu}^{a} \partial_{p}^{\nu}\right)\langle\delta f \delta f \delta f\rangle= & g Q_{a} p^{\mu} \partial_{\nu}^{p} \bar{f}\left\langle\left(\bar{D}_{\mu} a_{\nu}-\bar{D}_{\nu} a_{\mu}\right)^{a} \delta f \delta f\right\rangle-\langle\eta+\xi\rangle\langle\delta f \delta f\rangle \\
& +g p^{\mu} f^{a b c} Q_{c} \partial_{a}^{Q} \bar{f}\left\langle a_{b, \mu} \delta f \delta f\right\rangle+\langle(\eta+\xi) \delta f \delta f\rangle,
\end{aligned}
$$

and similarly for higher order correlators. Typically, the dynamical equations for correlators of $n$ fluctuations will couple to correlators ranging from the order $(n-1)$ up to order $(n+2)$ in the fluctuations. From cubic order onwards, the back-coupling contains terms non-linear in the correlation functions.

The dynamical equation for the energy momentum tensor of the gauge fields obtains from the average of (2.15). The corresponding one for the particles is found after integrating (2.14a) over $d P d Q p^{\mu}$. They read

$$
\begin{aligned}
\partial_{\nu} \bar{\Theta}^{\mu \nu}+\partial_{\nu}\left\langle\theta^{\mu \nu}\right\rangle & =-\bar{F}_{a}^{\mu \nu} \bar{J}_{\nu a}-\left\langle f_{a}^{\mu \nu} \delta J_{\nu a}\right\rangle-\left\langle f_{a}^{\mu \nu}\right\rangle \bar{J}_{\nu}^{a} \\
\partial_{\nu} \bar{t}^{\mu \nu} & =\bar{F}_{a}^{\mu \nu} \bar{J}_{\nu a}+\left\langle f_{a}^{\mu \nu} \delta J_{\nu a}\right\rangle+\left\langle f_{a}^{\mu \nu}\right\rangle \bar{J}_{\nu}^{a}
\end{aligned}
$$

such that the total energy momentum tensor is conserved.

Finally, the condition for the microscopic current conservation translates, after averaging, into two equations, one for the mean fields, and another one for the fluctuation fields. From $\left\langle D_{\mu} J^{\mu}\right\rangle=0$ we obtain

$$
\left(\bar{D}_{\mu} \bar{J}^{\mu}\right)_{a}+g f_{a b c}\left\langle a_{\mu}^{b} \delta J^{c, \mu}\right\rangle=0 .
$$

For the fluctuation current, we learn from $D_{\mu} J^{\mu}-\left\langle D_{\mu} J^{\mu}\right\rangle=0$ that

$$
\left(\bar{D}_{\mu} \delta J^{\mu}\right)_{a}+g f_{a b c}\left(a_{\mu}^{b} \bar{J}_{c}^{\mu}+a_{\mu}^{b} \delta J_{c}^{\mu}-\left\langle a_{\mu}^{b} \delta J_{c}^{\mu}\right\rangle\right)=0
$$

In sect. $\nabla B$, it is shown that (4.11) and (4.12) are consistent with the corresponding equations as obtained from the Yang-Mills equations.

\section{Second moment approximation and small coupling expansion}

The dynamical equations, as derived and presented here, are exact. No approximations have been performed. In order to solve them, it will be essential to apply some approximations, or to find a reasonable truncation for the hierarchy of dynamical equations for correlator functions. Here, we will indicate two approximation schemes, the second moment

${ }^{\dagger \dagger}$ The resulting hierarchy of dynamical equations for the correlators is very similar to the BBGKY hierarchy. While the BBGKY hierarchy links the dynamical equations for $n$-particle distribution functions with each other, here, we rather have a hierarchy for the correlator functions. 
approximation and the small coupling expansion. Although they have a distinct origin in the first place, we will see below (sect. V) that they are intimately linked due to the requirements of gauge invariance.

The so-called second moment approximation (sometimes also referred to as the polarization approximation [7]) is employed in order to simplify the dynamics of the fluctuations. It consists in equating

$$
\eta=\langle\eta\rangle, \quad \xi=\langle\xi\rangle, \quad J_{\text {fluc }}=\left\langle J_{\text {fluc }}\right\rangle .
$$

The essence of this approximation is that the dynamical equations for the correlators becomes homogeneous. It is easy to see that (4.7) or (4.8) depend only on quadratic or cubic correlators, respectively, once (4.13) is imposed. This approximation allows to cut the infinite hierarchy of equations down to a closed system of differential equations for both mean quantities and statistical fluctuations. The mean fields couple to quadratic correlators, and all correlators of degree $n$ couple amongst each others. This turns the dynamical equation for the fluctuations (4.6) into a differential equation linear in the fluctuations. This approximation is viable if the fluctuations and the two-particle correlators are small (see also sect. VI).

A seemingly different approximations concerns the non-Abelian sector of the theory. We shall perform a systematic perturbative expansion in powers of the gauge coupling $g$, keeping only the leading order terms. This can be done, because the differential operator appearing in the Boltzmann equation (2.14a) or (4.4a) admits such an expansion. In a small coupling expansion, the term $g Q_{a} \bar{F}_{\mu \nu}^{a} \partial_{p}^{\nu}$ is suppressed by a power in $g$ as compared to the leading order term $p^{\mu} \bar{D}_{\mu}$. Notice, that expanding the $g$ appearing within $p^{\mu} \bar{D}_{\mu}$ of (2.17) is not allowed as it will break gauge invariance. In this spirit, we expand as well

$$
\bar{f}=\bar{f}^{(0)}+g \bar{f}^{(1)}+g^{2} \bar{f}^{(2)}+\ldots
$$

and similarly for $\delta f$. This is at the basis for a systematic organization of the dynamical equations in powers of $g$. To leading order, this concerns in particular the cubic correlators in $\langle\eta\rangle$ and $\left\langle J_{\text {fluc }}\right\rangle$, which are suppressed by a power of $g$ as compared to the quadratic ones.

In principle, after these approximations are done, it should be possible to express the correlators of fluctuations appearing in (4.4) through known functions. This requires finding a solution of the fluctuation dynamics first.

\section{Collision integrals}

Let us comment on the interpretation of $\langle\eta\rangle$ and $\langle\xi\rangle$ as collision integrals of the macroscopic Boltzmann equation. The functions $\langle\eta\rangle$ and $\langle\xi\rangle$ appear only after the splitting (4.1) has been performed. This introduces new terms in the corresponding Boltzmann equation (4.4) for the mean fields, which are interpreted as effective collision integrals for the macroscopic transport equation. In this formalism, the collision integrals appear naturally as correlators of statistical fluctuations. The fluctuations in the gauge fields cause random changes in the motion of the particles, while random changes in the distribution function of 
the particles induce changes in the gauge fields. This is having the same effect as collisions, and yields a precise recipe as to obtain collision integrals from the microscopic theory.

In this light, the second moment approximation (4.13) can be interpreted as neglecting the back-coupling of collisions to the dynamics of the fluctuations. Also, neglecting cubic correlators appearing in $\langle\eta\rangle$ or $\left\langle J_{\text {fuc }}\right\rangle$ in favour of quadratic ones, to leading order in an expansion in the gauge coupling and for small fluctuations, is interpreted as neglecting the three-particle collisions in favour of two-particle collisions.

In order to find explicitly the corresponding collision integrals for the non-Abelian plasma, one has to solve first the dynamical equations for the fluctuations in the background of the mean fields. This step is interpreted as integrating-out the fluctuations. In general, this is a difficult task, in particular due to the non-linear terms present in (4.6). As argued above, this will only be possible when some approximations have been performed.

In the Abelian limit, (4.4) and (4.6) reduce to the known set of kinetic equations for Abelian plasmas [7]. In this limit, only the collision integral $\langle\eta\rangle$ survives. Here it is known that $\langle\eta\rangle$ can be expressed explicitly as the Balescu-Lenard collision integral, after solving the dynamical equations for the fluctuations and computing the correlators involved [7]. This proofs in a rigorous way the correspondence between fluctuations and collisions in an Abelian plasma.

\section{CONSISTENCY WITH GAUGE SYMMETRY}

In this section we shall discuss the consistency of the present approach with the requirements of the non-Abelian gauge symmetry. This discussion will concern the consistency of the general set of equations. The question of consistent approximations will be raised as well. In this section, we shall for convenience switch to a matrix notation, using the conventions $A \equiv A^{a} t_{a}, Q \equiv Q^{a} t_{a}$ etc., as well as $\left[t_{a}, t_{b}\right]=f_{a b c} t^{c}$ and $\operatorname{Tr} t_{a} t_{b}=-\frac{1}{2} \delta_{a b}$.

\section{A. Gauge covariance of the macroscopic equations}

As a consequence of the Wong equations being gauge invariant, we already established in sect. II that the microscopic set of equations (2.14) transforms covariantly under (finite) gauge transformations

$$
g A_{\mu}^{\prime}=U(x)\left(\partial_{\mu}+g A_{\mu}\right) U^{-1}(x), \quad U(x)=\exp -g \epsilon^{a}(x) t_{a},
$$

with parameter $\epsilon^{a}(x)$. We also have $f^{\prime}\left(x, p, Q^{\prime}\right)=f(x, p, Q)$, and

$$
Q^{\prime}=U(x) Q U^{-1}(x), \quad \partial_{Q}^{\prime}=U^{-1}(x) \partial_{Q} U(x), \quad F_{\mu \nu}^{\prime}=U(x) F_{\mu \nu} U^{-1}(x) .
$$

The question raises as to which extend this symmetry is conserved under the statistical average, performed when switching to a macroscopic description. This concerns in particular the subsequent split of the gauge field into a mean (or background) field, and a fluctuation field

$$
A_{\mu}=\bar{A}_{\mu}+a_{\mu}
$$


This separation is very similar to what is done in the background field method (BFM) [15]. Two symmetries are left after the splitting is performed, the background gauge symmetry,

$$
g \bar{A}_{\mu}^{\prime}=U(x)\left(\partial_{\mu}+g \bar{A}_{\mu}\right) U^{-1}(x), \quad a_{\mu}^{\prime}=U(x) a_{\mu} U^{-1}(x),
$$

and the fluctuation gauge symmetry,

$$
g \bar{A}_{\mu}^{\prime}=0, \quad g a_{\mu}^{\prime}=U(x)\left(\partial_{\mu}+g\left(\bar{A}_{\mu}+a_{\mu}\right)\right) U^{-1}(x) .
$$

Under the background gauge symmetry, the fluctuation field transforms covariantly (as a vector in the adjoint). In the first step, we will split (2.14a) according to (5.3). It follows trivially, that the resulting equation is invariant under both (5.4) and (5.5), if both $\bar{f}$ and $\delta f$ transform as $f$, that is, as a scalar.

The next step involves the statistical average. We require that the statistical average of the fluctuation vanishes, $\langle a\rangle=0$. This constraint is fully compatible with the background gauge symmetry, as $\langle a\rangle=0$ is invariant under (5.4). Any inhomogeneous transformation law for $a$, and in particular (5.5), can no longer be a symmetry of the macroscopic equations as the constraint $\langle a\rangle=0$ is not invariant

We rewrite now the transport equations in matrix convention. We have

$$
\begin{gathered}
p^{\mu}\left(\bar{D}_{\mu}+2 g \operatorname{Tr}\left(Q \bar{F}_{\mu \nu}\right) \partial_{p}^{\nu}\right) \bar{f}=\langle\eta\rangle+\langle\xi\rangle . \\
{\left[\bar{D}_{\mu}, \bar{F}^{\mu \nu}\right]+\left\langle J_{\text {fluc }}^{\nu}\right\rangle=\bar{J}^{\nu} .}
\end{gathered}
$$

with

$$
\begin{aligned}
\eta(x, p, Q) & =-2 g \operatorname{Tr}\left(Q f_{\mu \nu}\right) p^{\mu} \partial_{p}^{\nu} \delta f(x, p, Q) \\
\xi(x, p, Q) & =-2 g p^{\mu} \operatorname{Tr}\left(\left[Q, \partial^{Q}\right] a_{\mu}\right) \delta f(x, p, Q)-2 g^{2} \operatorname{Tr}\left(\left[a_{\mu}, a_{\nu}\right] Q\right) p^{\mu} \partial_{p}^{\nu} \bar{f}(x, p, Q) \\
J_{\text {fluc }}^{\nu}(x) & =g\left[\bar{D}^{\mu},\left[a_{\mu}, a^{\nu}\right]\right]+\left[a_{\mu},\left[\bar{D}^{\mu}, a^{\nu}\right]-\left[\bar{D}^{\nu}, a^{\mu}\right]\right]+g^{2}\left[a_{\mu},\left[a^{\mu}, a^{\nu}\right]\right] .
\end{aligned}
$$

and

$$
\begin{aligned}
p^{\mu}\left(\bar{D}_{\mu}-g \operatorname{Tr}\left(Q \bar{F}_{\mu \nu}\right) \partial_{p}^{\nu}\right) \delta f= & -2 g \operatorname{Tr}\left(Q\left[\bar{D}_{\mu}, a_{\nu}\right]-Q\left[\bar{D}_{\nu}, a_{\mu}\right]\right) p^{\mu} \partial_{\nu}^{p} \bar{f} \\
& -2 g p^{\mu} \operatorname{Tr}\left(\left[Q, \partial^{Q}\right] a_{\mu}\right) \bar{f}+\eta+\xi-\langle\eta+\xi\rangle, \\
{\left[\bar{D}_{\nu},\left[\bar{D}^{\nu}, a^{\mu}\right]\right]-\left[\bar{D}^{\mu},\left[\bar{D}_{\nu}, a^{\nu}\right]\right]+} & 2 g\left[\bar{F}^{\mu \nu}, a_{\nu}\right]+J_{\text {fluc }}^{\mu}-\left\langle J_{\text {fluc }}^{\mu}\right\rangle=\delta J^{\mu}
\end{aligned}
$$

It is now straightforward to realize that (5.6) - (5.8) transform covariantly under (5.4). It suffices to employ the cyclicity of the trace, and to note that $a_{\mu}$ and background covariant derivatives of it transform covariantly.

\footnotetext{
$\sharp$ This is similar to what happens in the BFM, where the fluctuation gauge symmetry can no longer be seen once the expectation value of the fluctuation field is set to zero. However, the symmetry (5.5) will be observed in both (4.4a) and (4.6a), as long as the terms linear in $\langle a\rangle$ are retained.
} 


\section{B. Consistent current conservation}

In (4.11) and (4.12), we have given the equations which imply the covariant current conservation of the mean and the fluctuation current. However, this information is contained both in the transport and in the Yang-Mills equation. It remains to be shown that these equations are self-consistent.

We start with the mean current $\bar{J}$. Performing $g \int d P d Q Q$ of the transport equation (5.6a), we find

$$
0=\left[\bar{D}_{\mu}, \bar{J}^{\mu}\right]+g\left\langle\left[a_{\mu}, \delta J^{\mu}\right]\right\rangle
$$

This is (4.11). Here, we used that

$$
\begin{aligned}
\int d P \eta(x, p, Q) & =0 \\
\int d P p^{\mu} F_{\mu \nu} \partial_{p}^{\nu} f(x, p, Q) & =0 \\
\int d P d Q Q \xi(x, p, Q) & =-\left[a_{\mu}, \delta J^{\mu}\right], \\
g \int d P d Q Q p^{\mu} \bar{D}_{\mu} \bar{f}(x, p, Q) & =\left[\bar{D}_{\mu}, \bar{J}^{\mu}\right] .
\end{aligned}
$$

Taking the background covariant derivative of (5.6b), we find

$$
0=\left[\bar{D}_{\mu}, \bar{J}^{\mu}\right]-\left[\bar{D}_{\mu},\left\langle J_{\text {fluc }}^{\mu}\right\rangle\right]
$$

which has to be consistent with (5.9). Thus, combining these two equations we end up with the consistency condition

$$
0=\left[\bar{D}_{\mu},\left\langle J_{\text {fluc }}^{\mu}\right\rangle\right]+g\left\langle\left[a_{\mu}, \delta J^{\mu}\right]\right\rangle .
$$

In the appendix $\mathrm{B}$, we established the identity

$$
0=\left[\bar{D}_{\mu}, J_{\text {fluc }}^{\mu}\right]+g\left[a_{\mu}, \delta J^{\mu}\right]+g\left[a_{\mu},\left\langle J_{\text {fluc }}^{\mu}\right\rangle\right]
$$

which follows using the explicit expressions for $\delta J^{\mu}$ from (5.8b) and for $J_{\text {fluc }}$ from (5.7c). Taking the average of (5.13) reduces it to (5.12), and establishes the self-consistent conservation of the mean current.

The analogous consistency equation for the fluctuation current obtains from (5.8a) after performing $g \int d P d Q Q$, and reads

$$
0=\left[\bar{D}_{\mu}, \delta J^{\mu}\right]+g\left[a_{\mu}, \delta J^{\mu}\right]+g\left[a_{\mu}, \bar{J}^{\mu}\right]-g\left\langle\left[a_{\mu}, \delta J^{\mu}\right]\right\rangle .
$$

This is (4.12). Here, in addition to (5.10), we made use of

$$
2 g \int d P d Q Q \operatorname{Tr}\left(\left[Q, \partial^{Q}\right] a_{\mu}\right) \bar{f}(x, p, Q)=g\left[a_{\mu}, \bar{J}^{\mu}\right] .
$$

The background covariant derivative of (5.8b) is given as 


$$
0=\left[\bar{D}_{\mu}, \delta J^{\mu}\right]+g\left[a_{\nu},\left[\bar{D}_{\mu}, \bar{F}^{\mu \nu}\right]\right]-\left[\bar{D}_{\mu}, J_{\text {fluc }}^{\mu}\right]+\left[\bar{D}_{\mu},\left\langle J_{\text {fluc }}^{\mu}\right\rangle\right] .
$$

Subtracting these equations yields the consistency condition

$$
0=\left[\bar{D}_{\mu}, J_{\text {fluc }}^{\mu}\right]+g\left[a_{\mu}, \delta J^{\mu}\right]-\left[\bar{D}_{\mu},\left\langle J_{\text {fluc }}^{\mu}\right\rangle\right]-g\left\langle\left[a_{\mu}, \delta J^{\mu}\right]\right\rangle+g\left[a_{\mu}, \bar{J}^{\mu}\right]-g\left[a_{\nu},\left[\bar{D}_{\mu}, \bar{F}^{\mu \nu}\right]\right] .
$$

Using (5.6b), (5.12) and (5.13) we confirm (5.17) explicitly. This establishes the selfconsistent conservation of the fluctuation current.

\section{Gauge-consistent approximations}

We close this section with a comment on the gauge consistency of approximate solutions. The consistent current conservation can no longer be taken for granted when it comes to finding approximate solutions of the equations. On the other hand, finding an explicit solution will require some type of approximations to be performed. The relevant question in this context is to know which approximations will be consistent with gauge invariance.

Consistency with gauge invariance requires that approximations have to be consistent with the background gauge symmetry. From the general discussion above we can already conclude that dropping any of the explicitly written terms in (4.4) - (4.6) is consistent with the background gauge symmetry (5.4). This holds in particular for the polarization or second moment approximation (4.13).

Consistency of the second moment approximation (4.13) with covariant current conservation turns out to be more restrictive. Employing $J_{\text {fluc }}=\left\langle J_{\text {fluc }}\right\rangle$ implies that (5.12) is only satisfied, if in addition

$$
0=\left[\bar{D}_{\nu},\left\langle\left[a_{\mu},\left[a^{\mu}, a^{\nu}\right]\right]\right\rangle\right]
$$

holds true. This is in accordance with neglecting cubic correlators for the collision integrals.

Similarly, the consistent conservation of the fluctuation current implies the consistency condition (5.17), and holds if

$$
0=\left[a_{\mu},\left\langle J_{\text {fluc }}^{\mu}\right\rangle\right]
$$

vanishes. It is interesting to note that the consistent current conservation relates the second moment approximation with the neglection of correlators of gauge field fluctuations. We conclude, that (4.13) with (5.18) and (5.19) form a gauge-consistent set of approximations.

\section{PHYSICAL SCALES: CLASSICAL VERSUS QUANTUM PLASMAS}

In the remaining part of the paper, we study the classical and quantum non-Abelian plasma close to equilibrium. Prior to this, we shall present a discussion of the relevant physical scales of both relativistic classical and quantum plasmas, close to equilibrium. We will restore here the fundamental constants $\hbar, c$ and $k_{B}$ in the formulas. 
To discuss the relevant physical scales in the classical non-Abelian plasma, it is convenient to discuss first the simpler Abelian case, which has been considered in detail in the literature [7]. At equilibrium the classical distribution function is given by the relativistic Maxwell distribution,

$$
\bar{f}^{\mathrm{eq}}\left(p_{0}\right)=A e^{-p_{0} / k_{B} T},
$$

where $A$ is a dimensionful constant which is fixed once the mean density of particles in the system is known. For massless particles $p_{0}=p c$, and if no further internal degrees of freedom are present, the mean density is given by $\bar{N}=8 \pi A\left(k_{B} T / c\right)^{3}$. The interparticle distance is then $\bar{r} \sim \bar{N}^{-1 / 3}$. As we are considering a classical plasma, we are assuming $\bar{r} \gg \lambda_{\mathrm{dB}}$, where $\lambda_{\mathrm{dB}}$ is the de Broglie wave length, $\lambda_{\mathrm{dB}} \sim \hbar / p$, with $p$ some typical momenta associated to the particles, thus $p \sim k_{B} T / c$. The previous inequality implies therefore $A \ll 1 / \hbar^{3}$, which is the condition under which quantum statistical effects can be neglected.

There is another typical scale in a plasma close to equilibrium, which is the Debye length $r_{D}$. The Debye length is the distance over which the screening effects of the electric fields in the plasma are felt. For an electromagnetic plasma, the Debye length squared is given by [7]

$$
r_{D}^{2}=k_{B} T / 4 \pi \bar{N} e^{2}
$$

Notice that the electric charge contained in the above formula is a dimensionful parameter: it is just the electric charge of the point particles of the system.

In the classical case, and in the absence of the fundamental constant $\hbar$, the only dimensionless quantity that can be constructed from the basic scales of the problem is the plasma parameter $\epsilon$. The plasma parameter is defined as the ratio [7]

$$
\epsilon=\bar{r}^{3} / r_{D}^{3}
$$

The quantity $1 / \epsilon$ gives the number of particles contained in a sphere of radius $r_{D}$. If $\epsilon \ll 1$ this implies that a large number of particles are in that sphere, and thus a large number of particles are interacting in this volume, and the collective character of their interactions in the plasma can not be neglected. For the kinetic description to make sense, $\epsilon$ has to be small [7]. This does not require, in general, that the interactions have to be weak and treated perturbatively.

Let us now consider the non-Abelian plasma. The interparticle distance is defined as in the previous case. The main differences with respect to the Abelian case concerns the Debye length, defined as the distance over which the screening effects of the non-Abelian electric fields in the plasma are noticed. It reads

$$
r_{D}^{2}=k_{B} T / 4 \pi \bar{N} g^{2} C_{2}
$$

where $C_{2}$, defined in (2.11), is a dimensionful quantity, carrying the same dimensions as the electric charge squared in (6.2). The coupling constant $g$ is a dimensionless parameter. In the non-Abelian plasma one can also construct the plasma parameter, defined as in (6.3). 
It is interesting to note that there are two natural dimensionless parameters in the nonAbelian plasma: $\epsilon$ and $g$. The condition for the plasma parameter being small translates into

$$
\left(\frac{4 \pi C_{2}}{k_{B} T}\right)^{3 / 2} \bar{N}^{1 / 2} g^{3} \ll 1,
$$

which is certainly satisfied for small gauge coupling constant $g \ll 1$. But it can also be fulfilled for a rarefied plasma. Thus, one may have a small plasma parameter without having a small gauge coupling constant. This is an interesting observation, since the inequalities $\epsilon \ll 1$ and $g \ll 1$ have different physical meanings. A small gauge coupling constant allows for treating the non-Abelian interaction perturbatively, while $\epsilon \ll 1$ just means having a collective field description of the physics occurring in the plasma. In principle, these two situations are different. If we knew how to treat the non-Abelian interactions exactly, we could also have a kinetic description of the classical non-Abelian plasmas without requiring $g \ll 1$.

Now we consider the quantum non-Abelian plasma, and consider the quantum counterparts of all the above quantities, as derived from quantum field theory. It is common to change the phase space measure $d^{3} x d^{3} p$ to the standard quantum normalization $d^{3} x d^{3} p /(2 \pi \hbar)^{3}$, which makes the quantum distribution function dimensionless. 3 For a quantum plasma at equilibrium the one particle distribution function is

$$
\bar{f}_{\mathrm{B}}^{\mathrm{eq}}\left(p_{0}\right)=\frac{1}{e^{p_{0} / k_{B} T}-1}, \quad \bar{f}_{\mathrm{F}}^{\mathrm{eq}}\left(p_{0}\right)=\frac{1}{e^{p_{0} / k_{B} T}+1}
$$

where the subscript $\mathrm{B} / \mathrm{F}$ refers to the bosonic/fermionic statistics. For a plasma of massless particles the mean density is $\bar{N} \sim\left(k_{B} T / \hbar c\right)^{3}$. The interparticle distance $\bar{r} \sim \bar{N}^{-1 / 3}$ becomes of the same order as the de Broglie wavelength, which is why quantum statistical effects can not be neglected in this case.

The value of the Debye mass is obtained from quantum field theory. It depends on the specific quantum statistics of the particles and their representation of $S U(N)$. From the quantum Debye mass one can deduce the value of the Debye length, which is of order

$$
r_{D}^{2} \sim \frac{1}{g^{2}}\left(\frac{\hbar c}{k_{B} T}\right)^{2} .
$$

It is not difficult to check that the plasma parameter, defined as in (6.3), becomes proportional to $g^{3}$. Thus $\epsilon$ is small if and only if $g \ll 1$. This is so, because in a quantum field theoretical formulation one does not have the freedom to fix the mean density $\bar{N}$ in an arbitrary way, as in the classical case. This explains why the kinetic description of a quantum non-Abelian plasma is deeply linked to the small gauge coupling regime of the theory.

\footnotetext{
$\S \S$ As done in the standard textbooks the factor $(2 \pi \hbar)^{3}$ is introduced into the measure, although some authors introduce it into the distribution function. We follow the first option.
} 


\section{THE CLASSICAL PLASMA CLOSE TO EQUILIBRIUM}

In this section we put the method to work for a hot non-Abelian plasma close to equilibrium. A prerequisite for a kinetic description to be viable is a small plasma parameter $\epsilon \ll 1$. We shall ensure this by imposing a small gauge coupling constant $g \ll 1$. Then, all further approximations as detailed in the sequel can be seen as a systematic expansion in powers of the gauge coupling.

\section{A. Non-Abelian Vlasov equations}

We begin with the set of mean field equations (4.4) and neglect the effect of statistical fluctuations entirely, $\delta f \equiv 0$. In that case, (4.4) becomes the non-Abelian Vlasov equations [5]

$$
\begin{aligned}
p^{\mu}\left(\bar{D}_{\mu}-g Q_{a} \bar{F}_{\mu \nu}^{a} \partial_{p}^{\nu}\right) \bar{f} & =0, \\
\bar{D}_{\mu} \bar{F}^{\mu \nu} & =\bar{J}^{\nu},
\end{aligned}
$$

where the colour current is given by

$$
\bar{J}_{a}^{\mu}(x)=g \sum_{\substack{\text { helicities } \\ \text { species }}} \int d P d Q Q_{a} p^{\mu} \bar{f}(x, p, Q),
$$

We will omit the species and helicity indices on the distribution functions, and in the sequel, we will also omit the above sum, in order to keep the notation as simple as possible. Equation (7.1a) is then solved perturbatively, as it admits a consistent expansion in powers of $g$. Close to equilibrium, we expand the distribution function as in (4.14) up to leading order in the coupling constant

$$
\bar{f}(x, p, Q)=\bar{f}^{\mathrm{eq}}\left(p_{0}\right)+g \bar{f}^{(1)}(x, p, Q) .
$$

In the strictly classical approach, the relativistic Maxwell distribution (6.1) at equilibrium is used. Here, we consider only massless particles, or massive particles with $m \ll T$, such that the masses can be neglected in a first approximation. We will consider also internal degrees of freedom, two helicities associated to every particle.

It is convenient to re-write the equations in terms of current densities. Consider the current densities

$$
\begin{aligned}
& J_{a_{1} \cdots a_{n}}^{\rho}(x, p)=g p^{\rho} \int d Q Q_{a_{1}} \cdots Q_{a_{n}} f(x, p, Q), \\
& \mathcal{J}_{a_{1} \cdots a_{n}}^{\rho}(x, v)=\int d \tilde{P} J_{a_{1} \cdots a_{n}}^{\rho}(x, p)
\end{aligned}
$$

Here, $v^{\mu}=(1, \mathbf{v})$ with $\mathbf{v}^{2}=1$. The measure $d \tilde{P}$ integrates over the radial components. It is related to (2.8) by $d P=d \tilde{P} d \Omega / 4 \pi$, and reads

$$
d \tilde{P}=4 \pi d p_{0} d|\mathbf{p}||\mathbf{p}|^{2} 2 \Theta\left(p_{0}\right) \delta\left(p^{2}\right)
$$


for massless particles. The colour current is obtained performing the remaining angle integration $J(x)=\int \frac{d \Omega}{4 \pi} \mathcal{J}(x, v)$. From now on we will omit the arguments of the current density $\mathcal{J}$, unless necessary to avoid confusion.

We now insert (7.2) into (7.1) and expand in powers of $g$. The leading order term $p \cdot D \bar{f}^{\text {eq. }}\left(p_{0}\right)$ vanishes. After multiplying (4.4a) by $g Q_{a} p^{\rho} / p_{0}$, summing over two helicities, and integrating over $d \tilde{P} d Q$, we obtain for the mean current density at order $g$

$$
\begin{aligned}
v^{\mu} \bar{D}_{\mu} \overline{\mathcal{J}}^{\rho}+m_{D}^{2} v^{\rho} v^{\mu} \bar{F}_{\mu 0} & =0, \\
\bar{D}_{\mu} \bar{F}^{\mu \nu} & =\bar{J}^{\nu},
\end{aligned}
$$

with the Debye mass

$$
m_{D}^{2}=-8 \pi g^{2} C_{2} \int_{0}^{\infty} d p p^{2} \frac{d \bar{f}^{\mathrm{eq}}(p)}{d p} .
$$

The solution to (7.5a) is now constructed with the knowledge of the retarded Green's function

$$
i v^{\mu} \bar{D}_{\mu} G_{\text {ret }}(x, y ; v)=\delta^{(4)}(x-y) .
$$

It reads

$$
G_{\mathrm{ret}}(x, y ; v)_{a b}=-i \theta\left(x_{0}-y_{0}\right) \delta^{(3)}\left(\mathbf{x}-\mathbf{y}-\mathbf{v}\left(x_{0}-y_{0}\right)\right) \bar{U}_{a b}(x, y),
$$

where $\bar{U}_{a b}$ is the parallel transporter obeying $\left.v^{\mu} \bar{D}_{\mu}^{x} \bar{U}_{a b}(x, y)\right|_{y=x-v t}=0$, and $\bar{U}_{a b}(x, x)=\delta_{a b}$. One finds

$$
\bar{J}_{a}^{\mu}(x)=-m_{D}^{2} \int \frac{d \Omega_{\mathbf{v}}}{4 \pi} \int_{0}^{\infty} d \tau \bar{U}_{a b}(x, x-v \tau) v^{\mu} v^{\nu} \bar{F}_{\nu 0, b}(x-v \tau)
$$

The above colour current agrees with the hard thermal loop (HTL) colour current «, [4], except for the value of the Debye mass.

From (7.5a) it is easy to estimate the typical momentum scale of the mean fields. If the effects of statistical fluctuations are neglected (and as we will see, this is equivalent to neglecting collisions), the typical momentum scales associated to the mean current and the mean field strength are of the order of the Debye mass $m_{D}$. We will refer to those scales as soft scales. The momentum scales with momenta $\ll m_{D}$ will be referred to as ultra-soft from now on.

\section{B. Leading order dynamics for mean fields and fluctuations}

We now allow for small statistical fluctuations $\delta f(x, p, Q)$ around (7.2), writing

$$
f(x, p, Q)=\bar{f}^{\mathrm{eq}}\left(p_{0}\right)+g \bar{f}^{(1)}(x, p, Q)+\delta f(x, p, Q)
$$

and re-write the approximations to (4.4) and (4.6) in terms of current densities and their fluctuations. Note that the fluctuations $\delta f(x, p, Q)$ in the close to equilibrium case are 
already of the order of $g$. This observation is important for the consistent approximation in powers of the gauge coupling. As a consequence, the term $g \bar{f}^{(1)}$ in $(7.10)$ will now account for the ultra-soft modes for momenta $\ll m_{D}$. Integrating-out the fluctuations results in an effective theory for the latter.

As before, we obtain the dynamical equation for the mean current density at leading order in $g$, after multiplying (4.4a) by $g Q_{a} p^{\rho} / p_{0}$, summing over two helicities, and integrating over $d \tilde{P} d Q$. The result is

$$
\begin{aligned}
v^{\mu} \bar{D}_{\mu} \overline{\mathcal{J}}^{\rho}+m_{D}^{2} v^{\rho} v^{\mu} \bar{F}_{\mu 0} & =\left\langle\eta^{\rho}\right\rangle+\left\langle\xi^{\rho}\right\rangle \\
\bar{D}_{\mu} \bar{F}^{\mu \nu}+\left\langle J_{\text {fluc }}^{\nu}\right\rangle & =\bar{J}^{\nu} .
\end{aligned}
$$

In a systematic expansion in $g$, we have to neglect cubic correlator terms as compared to quadratic ones, as they are suppressed explicitly by an additional power in $g$. Therefore, we find to leading order

$$
\begin{aligned}
\eta_{a}^{\rho} & =g \int d \tilde{P} \frac{p^{\rho}}{p_{0}}\left(\bar{D}_{\mu} a_{\nu}-\bar{D}_{\nu} a_{\mu}\right)^{b} \partial_{p}^{\nu} \delta J_{a b}^{\mu}(x, p), \\
\xi_{a}^{\rho} & =-g f_{a b c} v^{\mu} a_{\mu}^{b} \delta \mathcal{J}^{c, \rho}, \\
J_{\text {fluc }}^{\rho, a} & =g f^{d b c}\left(\bar{D}_{\mu}^{a d} a_{b}^{\mu} a_{c}^{\rho}+\delta^{a d} a_{\mu}^{b}\left(\bar{D}^{\mu} a^{\rho}-\bar{D}^{\rho} a^{\mu}\right)^{c}\right) .
\end{aligned}
$$

The same philosophy is applied to the dynamical equations for the fluctuations. To leading order in $g$, the result reads

$$
\begin{aligned}
& \left(v^{\mu} \bar{D}_{\mu} \delta \mathcal{J}^{\rho}\right)_{a}=-m_{D}^{2} v^{\rho} v^{\mu}\left(\bar{D}_{\mu} a_{0}-\bar{D}_{0} a_{\mu}\right)_{a}-g f_{a b c} v^{\mu} a_{\mu}^{b} \overline{\mathcal{J}}^{c, \rho}, \\
& v^{\mu}\left(\partial_{\mu} \delta_{a c} \delta_{b d}+g \bar{A}_{\mu}^{m}\left(f_{a m c} \delta_{b d}+f_{b m d} \delta_{a c}\right)\right) \delta \mathcal{J}_{c d}^{\rho}=g v^{\mu} a_{\mu}^{m}\left(f_{m a c} \delta_{b d}+f_{m b d} \delta_{a c}\right) \overline{\mathcal{J}}_{c d}^{\rho}, \\
& \left(\bar{D}^{2} a^{\mu}-\bar{D}^{\mu}(\bar{D} a)\right)_{a}+2 g f_{a b c} \bar{F}_{b}^{\mu \nu} a_{c, \nu}=\delta J_{a}^{\mu} .
\end{aligned}
$$

The typical momentum scale associated to the fluctuations can be estimated from (7.13). We find that it is of the order of the Debye mass $\sim m_{D}$, that is, of the same order as the mean fields in (7.5). This confirms explicitly the discussion made above. The typical momentum scales associated to the mean fields in (7.11) is therefore $\ll m_{D}$.

\section{Integrating-out the fluctuations}

We solve the equations for the fluctuations (7.13) with an initial boundary condition for $\delta f$, and $a_{\mu}(t=0)=0$. Exact solutions to (7.13a) and (7.13b) can be obtained.

Let us start by solving the homogeneous differential equation

$$
v^{\mu} \bar{D}_{\mu} \delta \mathcal{J}^{\rho}=0
$$

with the initial condition $\delta \mathcal{J}_{a}^{\mu}(t=0, \mathbf{x}, v)$. It is not difficult to check, by direct inspection, that the solution to the homogeneous problem is

$$
\delta \mathcal{J}_{a}^{\rho}(x, v)=\bar{U}_{a b}(x, x-v t) \delta \mathcal{J}_{b}^{\rho}(t=0, \mathbf{x}-\mathbf{v} t, v)
$$


The solution of (7.13a) is now constructed using the retarded Green's function (7.8). For $x_{0} \equiv t \geq 0$ the complete solution can be expressed as

$$
\begin{aligned}
\delta \mathcal{J}_{a}^{\rho}(x, v)= & -\int_{0}^{\infty} d \tau \bar{U}_{a b}\left(x, x_{\tau}\right)\left(m_{D}^{2} v^{\rho} v^{\mu}\left(\bar{D}_{\mu} a_{0}-\bar{D}_{0} a_{\mu}\right)^{b}\left(x_{\tau}\right)+g f_{b d c} v^{\mu} a_{\mu}^{d}\left(x_{\tau}\right) \overline{\mathcal{J}}_{c}^{\rho}\left(x_{\tau}, v\right)\right) \\
& +\bar{U}_{a b}\left(x, x_{t}\right) \delta \mathcal{J}_{b}^{\rho}\left(x_{t}, v\right) .
\end{aligned}
$$

We have introduced $x_{\tau} \equiv x-v \tau$, and thus $x_{t}=(0, \mathbf{x}-\mathbf{v} t)$. Since $a_{\mu}(t=0)=0$, one can check that the above current obeys the correct initial condition.

The equation (7.13b) can be solved in a similar way. The solution is

$$
\begin{aligned}
\delta \mathcal{J}_{a b}^{\rho}(x, v)= & \bar{U}_{a m}\left(x, x_{t}\right) \bar{U}_{b n}\left(x, x_{t}\right) \delta \mathcal{J}_{m n}^{\rho}\left(x_{t}, v\right) \\
& -g \int_{0}^{\infty} d \tau \bar{U}_{a m}\left(x, x_{\tau}\right) \bar{U}_{b n}\left(x, x_{\tau}\right)\left(f_{m p c} \delta_{n d}+f_{n p d} \delta_{m c}\right) v^{\mu} a_{\mu}^{p}\left(x_{\tau}\right) \overline{\mathcal{J}}_{c d}^{\rho}\left(x_{\tau}, v\right) .
\end{aligned}
$$

Now we seek for solutions to the equation (7.13c) with the colour current of the fluctuation as found above. However, notice that this equation is non-local in $a_{\mu}$, which makes it difficult to find exact solutions. Nevertheless, one can solve the equation in an iterative way, by making a double expansion in both $g \bar{A}$ and $g \overline{\mathcal{J}}$. This is possible since the parallel transporter $\bar{U}$ admits an expansion in $g \bar{A}$, so that the current $\delta \mathcal{J}^{\rho}$ can be expressed as a power series in $g \bar{A}$

$$
\delta \mathcal{J}^{\rho}=\delta \mathcal{J}^{\rho(0)}+\delta \mathcal{J}^{\rho(1)}+\delta \mathcal{J}^{\rho(2)}+\cdots
$$

and thus $(\overline{7.13 \mathrm{~d}})$ can be solved for every order in $g \bar{A}$. To lowest order in $g \bar{A}$, using $\bar{U}_{a b}=$ $\delta_{a b}+\mathcal{O}(g \bar{A})$, equation $\left.7.13 \mathrm{~d}\right)$ becomes

$$
\partial^{\mu}\left(\partial_{\mu} a_{\nu, a}^{(0)}-\partial_{\nu} a_{\mu, a}^{(0)}\right)=\delta J_{\nu, a}^{(0)}
$$

Using the one-sided Fourier transform [7], and (7.16), we find

$$
\begin{aligned}
\delta J_{a+}^{\mu(0)}(k)= & \Pi_{a b}^{\mu \nu}(k) a_{\nu, b}^{(0)}(k)-g f_{a b c} \int \frac{d \Omega_{\mathbf{v}}}{4 \pi} \frac{1}{-i k \cdot v} \int \frac{d^{4} q}{(2 \pi)^{4}} v^{\rho} a_{\rho}^{b(0)}(q) \overline{\mathcal{J}}^{\mu, c}(k-q, v) \\
& +\int \frac{d \Omega_{\mathbf{v}}}{4 \pi} \frac{\delta \mathcal{J}_{a}^{\mu}(t=0, \mathbf{k}, v)}{-i k \cdot v},
\end{aligned}
$$

where $\Pi_{a b}^{\mu \nu}(k)$ is the polarization tensor in the plasma, which reads

$$
\Pi_{a b}^{\mu \nu}(k)=\delta_{a b} m_{D}^{2}\left(-g^{\mu 0} g^{\nu 0}+k_{0} \int \frac{d \Omega_{\mathbf{v}}}{4 \pi} \frac{v^{\mu} v^{\nu}}{k \cdot v}\right),
$$

and agrees with the HTL polarization tensor of QCD [4,6], except in the value of the Debye mass. Retarded boundary conditions are assumed above, with the prescription $k_{0} \rightarrow k_{0}+i 0^{+}$.

We solve (7.19) iteratively in momentum space for $a_{\mu}$ as an infinite power series in $g \overline{\mathcal{J}}$,

$$
a_{\mu}^{(0)}=a_{\mu}^{(0,0)}+a_{\mu}^{(0,1)}+a_{\mu}^{(0,2)}+\ldots
$$


where the second index counts the powers of the background current $g \overline{\mathcal{J}}$. Notice that in this type of Abelianized approximation, the equation (7.19) has a (perturbative) Abelian gauge symmetry associated to the fluctuation $a_{\mu}$. This symmetry is only broken by the term proportional to $\overline{\mathcal{J}}$ in the current. It is an exact symmetry for the term $a_{\mu}^{(0,0)}$ in the above expansion. We will use this perturbative gauge symmetry in order to simplify the computations, and finally check that the results of the approximate collision integrals do not depend on the choice of the fluctuation gauge.

Using the one-sided Fourier transform, we find the following results for the longitudinal fields, in the gauge $\mathbf{k} \cdot \mathbf{a}^{(0,0)}=0$,

$$
\begin{aligned}
& a_{0, a+}^{(0,0)}(k)=\frac{1}{\mathbf{k}^{2}-\Pi_{L}} \int \frac{d \Omega_{\mathbf{v}}}{4 \pi} \frac{\delta \mathcal{J}_{0, a}(t=0, \mathbf{k}, v)}{-i k \cdot v}, \\
& a_{0, a+}^{(0,1)}(k)=\frac{-g f_{a b c}}{\mathbf{k}^{2}-\Pi_{L}} \int \frac{d \Omega_{\mathbf{v}}}{4 \pi} \frac{1}{-i k \cdot v} \int \frac{d^{4} q}{(2 \pi)^{4}} v^{\mu} a_{\mu}^{b(0,0)}(q) \overline{\mathcal{J}}_{0}^{c}(k-q, v),
\end{aligned}
$$

while we find

$$
\begin{aligned}
& a_{i, a+}^{T(0,0)}(k)=\frac{1}{-k^{2}+\Pi_{T}} \int \frac{d \Omega_{\mathbf{v}}}{4 \pi} \frac{\delta \mathcal{J}_{i, a}^{T}(t=0, \mathbf{k}, v)}{-i k \cdot v}, \\
& a_{i, a+}^{T(0,1)}(k)=\frac{-g f_{a b c}}{-k^{2}+\Pi_{T}} P_{i j}^{T}(\mathbf{k}) \int \frac{d \Omega_{\mathbf{v}}}{4 \pi} \frac{1}{-i k \cdot v} \int \frac{d^{4} q}{(2 \pi)^{4}} v^{\mu} a_{\mu}^{b(0,0)}(q) \overline{\mathcal{J}}_{j}^{c}(k-q, v),
\end{aligned}
$$

for the transverse fields. .** $^{* * *}$ The functions $\Pi_{L / T}(k)$ are the longitudinal/transverse polarization tensor of the plasma, $P_{i j}^{T}(\mathbf{k})=\delta_{i j}-k_{i} k_{j} / \mathbf{k}^{2}$ the transverse projector, and $a_{i}^{T} \equiv P_{i j}^{T} a_{j}$.

In the approximation $g \ll 1$, it will be enough to consider the solution of leading (zeroth) order in $g \bar{A}$, and the zeroth and first order in $g \overline{\mathcal{J}}$. The remaining terms are subleading in the leading logarithmic approximation. However, notice that we have all the tools necessary to compute the complete (perturbative) series. If we could solve equation (7.13d) exactly, it would not be necessary to use this perturbative expansion.

\section{The statistical correlator of fluctuations}

With the explicit expressions obtained in (7.20), (7.23) and (7.24), we can express all fluctuations in terms of initial conditions $\delta \mathcal{J}_{a}^{\mu}(t=0, \mathbf{x}, v)$ and the mean fields.

In order to compute the correlator of initial conditions, we will make use of the result obtained in sect. III. For each species of particles or internal degree of freedom, the statistical average over initial conditions can be expressed as

$$
\begin{aligned}
\left\langle\delta f(t=0, \mathbf{x}, p, Q) \delta f\left(t=0, \mathbf{x}^{\prime}, p^{\prime}, Q^{\prime}\right)\right\rangle= & \delta^{(3)}\left(\mathbf{x}-\mathbf{x}^{\prime}\right) \delta^{(3)}\left(\mathbf{p}-\mathbf{p}^{\prime}\right) \delta\left(Q-Q^{\prime}\right) \bar{f}(x, p, Q) \\
& +\tilde{g}_{2}\left(\mathbf{x}, p, Q ; \mathbf{x}^{\prime}, p^{\prime}, Q^{\prime}\right)
\end{aligned}
$$

${ }^{* * *}$ In [4], we used a more condensed notation. There, the functions $a_{i,+}^{(0,0)}$ and $a_{i,+}^{(0,1)}$ have been denoted $a_{i,+}^{(0)}$ and $a_{i,+}^{(1)}$. 
where the function $\tilde{g}_{2}$ obtains from the two-particle correlator, and

$$
\delta\left(Q-Q^{\prime}\right)=\frac{1}{c_{R}} \delta\left(\boldsymbol{\phi}-\boldsymbol{\phi}^{\prime}\right) \delta(\boldsymbol{\pi}-\boldsymbol{\pi})
$$

and $\phi, \pi$ are the Darboux variables associated to the colour charges $Q_{a}$. The appearance of the factor $1 / c_{R}$ in the above expression is due to the change of normalization factors associated to the functions $\mathcal{N}$ and $f$, as we mentioned at the end of sect. III. The above statistical average is all we need to evaluate the collision integrals below.

From (7.25) one deduces the statistical average over colour current densities $\delta \mathcal{J}$. We expand the momentum $\delta$-function in polar coordinates

$$
\delta^{(3)}\left(\mathbf{p}-\mathbf{p}^{\prime}\right)=\frac{1}{p^{2}} \delta\left(p-p^{\prime}\right) \delta^{(2)}\left(\Omega_{\mathbf{v}}-\Omega_{\mathbf{v}^{\prime}}\right),
$$

where $\Omega_{\mathbf{v}}$ represents the angular variables associated to the vector $\mathbf{v}=\mathbf{p} /|\mathbf{p}|$. After simple integrations we arrive at

$$
\begin{aligned}
\left\langle\delta \mathcal{J}_{\mu}^{a}(t=0, \mathbf{x}, v) \delta \mathcal{J}_{\nu}^{b}\left(t=0, \mathbf{x}^{\prime}, v^{\prime}\right)\right\rangle= & 2 g^{2} B_{C} C_{2} \delta^{a b} v_{\mu} v_{\nu}^{\prime} \delta^{(3)}\left(\mathbf{x}-\mathbf{x}^{\prime}\right) \delta^{(2)}\left(\Omega_{\mathbf{v}}-\Omega_{\mathbf{v}^{\prime}}\right) \\
& +\tilde{g}_{2, \mu \nu}^{a b}\left(\mathbf{x}, v ; \mathbf{x}^{\prime}, v^{\prime}\right)
\end{aligned}
$$

where $v^{\mu}=(1, \mathbf{v})$, and

$$
B_{C}=16 \pi^{2} \int_{0}^{\infty} d p p^{2} \bar{f}^{\mathrm{eq}}(p)
$$

The function $\tilde{g}_{2, \mu \nu}^{a b}$ is obtained from the two-particle correlation function $\tilde{g}_{2}$. Notice that we have neglected the piece $g \bar{f}^{(1)}$ above, as this is subleading in an expansion in $g$.

Since we know the dynamical evolution of all fluctuations we can also deduce the dynamical evolution of the correlators of fluctuations, with the initial condition (7.25). This corresponds to solving (4.7) in the present approximation. It is convenient to proceed as follows [7]. We separate the colour current (7.16) into a source part and an induced part,

$$
\delta \mathcal{J}^{\mu}=\delta \mathcal{J}_{\text {source }}^{\mu}+\delta \mathcal{J}_{\text {induced }}^{\mu} .
$$

The induced piece $\delta \mathcal{J}_{\text {induced }}^{\mu}$ is the part of the current which contains the dependence on $a_{\mu}$, and thus takes the polarization effects of the plasma into account. The source piece $\delta \mathcal{J}_{\text {source }}^{\mu}$ is the part of the current which depends only on the initial condition, given by the solution of the homogeneous equation (7.14). This splitting will be useful since ultimately all the relevant correlators can be expressed in terms of correlators of $\delta \mathcal{J}_{\text {source }}^{\mu}$.

From the explicit solution (7.16) and the average (7.28) we then find, at leading order in $g$ and neglecting the non-local term in (7.28)

$$
\begin{gathered}
\left\langle\delta \mathcal{J}_{\text {source }}^{a, \mu}(x, v) \delta \mathcal{J}_{\text {source }}^{b, \nu}\left(x^{\prime}, v^{\prime}\right)\right\rangle=2 g^{2} B_{C} C_{2} v^{\mu} v^{\prime \nu} \delta^{(3)}\left(\mathbf{x}-\mathbf{x}^{\prime}-\mathbf{v}\left(t-t^{\prime}\right)\right) \delta^{(2)}\left(\Omega_{\mathbf{v}}-\Omega_{\mathbf{v}^{\prime}}\right) \\
\times \bar{U}^{a c}(x, x-v t) \bar{U}^{b c}\left(x^{\prime}, x^{\prime}-v^{\prime} t^{\prime}\right)
\end{gathered}
$$

Here, and from now on, we neglect the non-local piece $\tilde{g}_{2, \mu \nu}^{a b}$. It can be shown [7] that they give contributions which decrease rapidly with time, so that for asymptotic large times, they vanish. 
Expanding the parallel transporter $\bar{U}$, and switching to momentum space we find the spectral density to zeroth order in $g \bar{A}$

$$
\left\langle\delta \mathcal{J}_{\mu}^{a} \delta \mathcal{J}_{\nu}^{b}\right\rangle_{k, v, v^{\prime}}^{\text {source }(0)}=2 g^{2} B_{C} C_{2} \delta^{a b} v_{\mu} v_{\nu}^{\prime} \delta^{(2)}\left(\Omega_{\mathbf{v}}-\Omega_{\mathbf{v}^{\prime}}\right)(2 \pi) \delta(k \cdot v) .
$$

As an illustrative example, let us compute the correlator of two transverse fields $a$. Using $(7.24 \mathrm{a})$ and $(7.32)$ one arrives at

$$
\left\langle a_{i, a}^{T(0,0)}(k) a_{j, b}^{T(0,0)}(q)\right\rangle=g^{2} B_{C} C_{2} \delta_{a b}(2 \pi)^{4} \delta^{(4)}(k+q) \frac{P_{i k}^{T}(\mathbf{k}) P_{j l}^{T}(\mathbf{k})}{\left|-k^{2}+\Pi_{T}\right|^{2}} \int \frac{d \Omega_{\mathbf{v}}}{4 \pi} v_{k} v_{l} \delta(k \cdot v) .
$$

Since the imaginary part of the polarization tensor, which describes Landau damping, can be expressed as [6]

$$
\operatorname{Im} \Pi_{a b}^{\mu \nu}(k)=-\delta_{a b} m_{D}^{2} \pi k_{0} \int \frac{d \Omega_{\mathbf{v}}}{4 \pi} v^{\mu} v^{\nu} \delta(k \cdot v),
$$

the statistical correlator can finally be written as

$$
\left\langle a_{i, a}^{T(0,0)}(k) a_{j, b}^{T(0,0)}(q)\right\rangle=\frac{4 \pi T}{k_{0}} \frac{\operatorname{Im} \prod_{i j, T}^{a b}(k)}{\left|-k^{2}+\Pi_{T}\right|^{2}}(2 \pi)^{3} \delta^{(4)}(k+q) .
$$

Here, we have used the relation

$$
2 g^{2} C_{2} B_{C}=4 \pi T m_{D}^{2} .
$$

Equation (7.35) is a form of the fluctuation dissipation theorem (FDT), which links the dissipative processes occurring in the plasma with statistical fluctuations.

\section{E. The collision integral}

We are now ready to compute at leading order in $g$ the collision integrals appearing on the r.h.s. of (7.11a). We shall combine the expansions introduced earlier to expand the collision integrals in powers of $\overline{\mathcal{J}}$ (while retaining only the zeroth order in $g \bar{A}$ ),

$$
\langle\xi\rangle=\left\langle\xi^{(0)}\right\rangle+\left\langle\xi^{(1)}\right\rangle+\left\langle\xi^{(2)}\right\rangle+\ldots,
$$

and similarly for $\langle\eta\rangle$ and $\left\langle J_{\text {fuc }}\right\rangle$. We find that the induced current $\left\langle J_{\text {fluc }}^{(0)}\right\rangle$ vanishes, as do the fluctuation integrals $\left\langle\eta^{(0)}\right\rangle$ and $\left\langle\xi^{(0)}\right\rangle$. The vanishing of $\left\langle J_{\text {fuc }}^{(0)}\right\rangle$ is deduced trivially from the fact that $\left\langle a_{a}^{(0,0)} a_{b}^{(0,0)}\right\rangle \sim \delta_{a b}$, while this correlator always appears contracted with the antisymmetric constants $f_{a b c}$ in $J_{\text {fluc }}$. To check that $\left\langle\eta^{(0)}\right\rangle=0$, one needs the statistical correlator $\left\langle\delta J_{a}^{\mu} \delta J_{a b}^{\rho}\right\rangle$, which is proportional to $\sum_{a} d_{a a b}=0$ for $S U(N)$. The vanishing of $\left\langle\eta^{(0)}\right\rangle$ is consistent with the fact that in the Abelian limit the counterpart of $\langle\eta\rangle$ vanishes at equilibrium [0]. Finally, $\left\langle\xi^{(0)}\right\rangle=0$ due to a contraction of $f_{a b c}$ with a correlator symmetric in the colour indices. 
In the same spirit we evaluate the terms in the collision integrals containing one $\overline{\mathcal{J}}$ field and no background gauge $\bar{A}$ fields. Consider

$$
\begin{aligned}
\left\langle\xi_{\rho, a}^{(1)}\right\rangle=g f_{a b c} v^{\mu}\{ & -\left\langle a_{\mu, b}^{(0,1)}(x) \delta \mathcal{J}_{\rho, c}^{(0)}(x, v)\right\rangle \\
& \left.+g f_{c d e} v^{\nu} \int_{0}^{\infty} d \tau \overline{\mathcal{J}}_{\rho, e}\left(x_{\tau}, v\right)\left\langle a_{\mu, b}^{(0,0)}(x) a_{\nu, d}^{(0,0)}\left(x_{\tau}\right)\right\rangle\right\} .
\end{aligned}
$$

Using the values for $a_{\mu}$ and $\delta \mathcal{J}^{(0)}$ as found earlier, we obtain in momentum space

$$
\left\langle\xi_{\rho, a}^{(1)}(k, v)\right\rangle \approx-g^{4} C_{2} N B_{C} v_{\rho} \int \frac{d \Omega_{\mathbf{v}^{\prime}}}{4 \pi} C\left(\mathbf{v}, \mathbf{v}^{\prime}\right)\left(\overline{\mathcal{J}}_{a}^{0}(k, v)-\overline{\mathcal{J}}_{a}^{0}\left(k, v^{\prime}\right)\right)
$$

with

$$
C\left(\mathbf{v}, \mathbf{v}^{\prime}\right)=\int \frac{d^{4} q}{(2 \pi)^{4}}\left|\frac{v_{i} P_{i j}^{T}(q) v_{j}^{\prime}}{-q^{2}+\Pi_{T}}\right|^{2}(2 \pi) \delta(q \cdot v)(2 \pi) \delta\left(q \cdot v^{\prime}\right)
$$

Here, the symbol $\approx$ means that only the leading terms have been retained. To arrive at the above expression we have used the $S U(N)$ relation $f_{a b c} f_{a b d}=N \delta_{c d}$. Within the momentum integral, we have neglected in the momenta of the mean fields, $k$, in front of the momenta of the fluctuations, $q$. As we discussed above, the momenta associated to the background fields are much smaller than those associated to the fluctuations which justifies this approximation to leading order. This is precisely what makes the collision integral, which in principle contains a convolution over momenta, local in $k$-space (resp. $x$-space). The only remaining non-locality stems from the angle convolution of $(\overline{7.40})$. Notice that we have only written the part arising from the transverse fields $a$, as the one associated to the longitudinal modes is subleading. This is easy to see once one realizes that the above integral is logarithmic divergent in the infrared (IR) region, while the longitudinal contribution is finite. At this point, we can also note that the collision integral computed this way is independent on the (perturbative) Abelian gauge used to solve equation (7.19). This is so because the collision integral computed this way can always be expressed in terms of the imaginary parts of the polarization tensors (7.34) in the plasma, which are known to be gauge-independent.

The integral (7.40) has also been obtained in [13], on the basis of a phenomenological derivation of the Boltzmann collision integral for a quantum plasma. The only difference consists in the value of the Debye mass appearing in the polarization tensor.

In any case, the transverse polarization tensor $\Pi_{T}$ vanishes at $q_{0}=0$, and the dynamical screening is not enough to make (7.40) finite. An IR cutoff must be introduced by hand in order to evaluate the integral. With a cutoff of order $g m_{D}$ we thus find at logarithmic accuracy

$$
C\left(\mathbf{v}, \mathbf{v}^{\prime}\right) \approx \frac{2}{\pi^{2} m_{D}^{2}} \ln (1 / g) \frac{\left(\mathbf{v} \cdot \mathbf{v}^{\prime}\right)^{2}}{\sqrt{1-\left(\mathbf{v} \cdot \mathbf{v}^{\prime}\right)^{2}}}
$$

Using also the relation $(\mathbf{7 . 3 6})$ we finally arrive at the collision integral to leading logarithmic accuracy, 


$$
\begin{aligned}
\left\langle\xi_{\rho, a}^{(1)}(x, v)\right\rangle & =-\frac{g^{2}}{4 \pi} N T \ln (1 / g) v_{\rho} \int \frac{d \Omega_{\mathbf{v}^{\prime}}}{4 \pi} \mathcal{I}\left(v, v^{\prime}\right) \overline{\mathcal{J}}_{a}^{0}\left(x, v^{\prime}\right) \\
\mathcal{I}\left(\mathbf{v}, \mathbf{v}^{\prime}\right) & =\delta^{(2)}\left(\mathbf{v}-\mathbf{v}^{\prime}\right)-\mathcal{K}\left(\mathbf{v}, \mathbf{v}^{\prime}\right), \quad \mathcal{K}\left(\mathbf{v}, \mathbf{v}^{\prime}\right)=\frac{4}{\pi} \frac{\left(\mathbf{v} \cdot \mathbf{v}^{\prime}\right)^{2}}{\sqrt{1-\left(\mathbf{v} \cdot \mathbf{v}^{\prime}\right)^{2}}}
\end{aligned}
$$

where we have introduced $\delta^{(2)}\left(\mathbf{v}-\mathbf{v}^{\prime}\right) \equiv 4 \pi \delta^{(2)}\left(\Omega_{\mathbf{v}}-\Omega_{\mathbf{v}}^{\prime}\right), \int \frac{d \Omega_{\mathbf{v}}}{4 \pi} \delta^{(2)}\left(\mathbf{v}-\mathbf{v}^{\prime}\right)=1$.

We can verify explicitly that the collision integral to leading logarithmic accuracy is consistent with gauge invariance. This should be so, as the approximations employed have been shown in sect. $\mathrm{VQ}$ on general grounds to be consistent with gauge invariance. Evaluating the correlator in (4.11) in the leading logarithmic approximation yields

$$
g f_{a b c}\left\langle a_{\mu}^{b}(x) \delta J_{c}^{\mu}(x)\right\rangle=-\frac{g^{2}}{4 \pi} N T \ln (1 / g) \int \frac{d \Omega_{\mathbf{v}}}{4 \pi} \frac{d \Omega_{\mathbf{v}^{\prime}}}{4 \pi} \mathcal{I}\left(\mathbf{v}, \mathbf{v}^{\prime}\right) \overline{\mathcal{J}}_{a}^{0}\left(x, v^{\prime}\right)
$$

which vanishes, because

$$
\int \frac{d \Omega_{\mathbf{v}}}{4 \pi} \mathcal{I}\left(\mathbf{v}, \mathbf{v}^{\prime}\right)=0
$$

We thus establish that $\bar{D}_{\mu} \bar{J}^{\mu}=0$, in accordance with (7.11b) in the present approximation.

\section{F. The source for stochastic noise}

The collision integral obtained above describes a dissipative process in the plasma, so in principle, it could trigger the system to abandon equilibrium. Whenever dissipative processes are encountered, it is important to identify as well the stochastic source related to it. This is the essence of the fluctuation-dissipation theorem (FDT). Phenomenologically, this is well known, and sometimes used the other way around: imposing the FDT allows to add by hand a source for stochastic noise with the strength of its self-correlator fixed by the dissipative processes.

In the present formalism, we are able to identify directly the source for stochastic noise which prevents the system from abandoning equilibrium. This proves, that the FDT does hold (analogous considerations have been presented in [12]). The relevant noise term is given by the contributions from the transversal gauge fields in $\xi^{(0)}$. While its average vanishes, $\left\langle\xi^{(0)}\right\rangle=0$, its correlator

$$
\left\langle\xi_{a}^{\rho(0)}(x, v) \xi_{b}^{\sigma(0)}\left(y, v^{\prime}\right)\right\rangle=g^{2} f_{a p c} f_{b d e} v^{\mu} v^{\prime \nu}\left\langle a_{\mu}^{p}(x) \delta \mathcal{J}_{\text {source }}^{\rho, c}(x, v) a_{\nu}^{d}(y) \delta \mathcal{J}_{\text {source }}^{\sigma, e}\left(y, v^{\prime}\right)\right\rangle^{(0)}
$$

does not. In order to evaluate this correlator we switch to Fourier space. Within the second moment approximation we expand the correlator $\langle\delta f \delta f \delta f \delta f\rangle$ into products of second order correlators $\langle\delta f \delta f\rangle\langle\delta f \delta f\rangle$ and find

$$
\begin{aligned}
\left\langle\xi_{a}^{\rho(0)}(k, v) \xi_{b}^{\sigma(0)}\left(p, v^{\prime}\right)\right\rangle= & g^{2} f_{a p c} f_{b d e} v^{\mu} v^{\prime \nu} \int \frac{d^{4} q}{(2 \pi)^{4}} \int \frac{d^{4} r}{(2 \pi)^{4}} \\
& \left\{\left\langle a_{\mu p}^{(0,0)}(q) a_{\nu d}^{(0,0)}(r)\right\rangle\left\langle\delta \mathcal{J}_{\text {source }}^{(0), c}(k-q, v) \delta \mathcal{J}_{\text {source }}^{(0) \sigma, e}\left(p-r, v^{\prime}\right)\right\rangle\right. \\
& \left.+\left\langle a_{\mu p}^{(0,0)}(q) \delta \mathcal{J}_{\text {source }}^{(0) \sigma, e}\left(p-r, v^{\prime}\right)\right\rangle\left\langle\delta \mathcal{J}_{\text {source }}^{(0) \rho, c}(k-q, v) a_{\nu d}^{(0,0)}(r)\right\rangle\right\}
\end{aligned}
$$


In the leading logarithmic approximation we retain only the contributions from the transverse modes. Evaluating the correlators leads to

$$
\left\langle\xi_{(0)}^{\mu, a}(x, v) \xi_{(0)}^{\nu, b}\left(y, v^{\prime}\right)\right\rangle=\frac{g^{6} N C_{2}^{2} B_{C}^{2}}{(2 \pi)^{3} m_{D}^{2}} \ln (1 / g) v^{\mu} v^{\prime \nu} \mathcal{I}\left(\mathbf{v}, \mathbf{v}^{\prime}\right) \delta^{a b} \delta^{(4)}(x-y) .
$$

After averaging over the angles of $\mathbf{v}$ and $\mathbf{v}^{\prime}$, and using the relation (7.36), the correlator becomes

$$
\left\langle\xi_{(0)}^{i, a}(x) \xi_{(0)}^{j, b}(y)\right\rangle=2 T \frac{m_{D}^{2}}{3} \frac{g^{2}}{4 \pi} N T \ln (1 / g) \delta^{a b} \delta^{i j} \delta^{(4)}(x-y)
$$

In particular, all correlators $\left\langle\xi_{(0)}^{0}(x) \xi_{(0)}^{\mu}(y)\right\rangle$ vanish. Equation $(7.49)$ identifies $\xi_{(0)}^{i}(x)$ as a source of white noise.

The presence of this noise term does not interfere with the covariant current conservation confirmed at the end of the previous section. This can be seen as follows. The noise

term enters (7.44) as the angle average over the 0 -component of $\xi_{(0)}^{\mu}(x, v)$. As we have established above, the logarithmically enhanced contribution from the noise source stems from its correlator (7.48). Averaging the temporal component of (7.48) over the angles of $\mathbf{v}$, and using (7.45), it follows that

$$
\left\langle\xi_{(0)}^{0, a}\left(x, v^{\prime}\right) \int \frac{d \Omega_{\mathbf{v}}}{4 \pi} \xi_{(0)}^{0, b}(y, v)\right\rangle=0
$$

We thus conclude, that the temporal component of the noise, $\xi_{(0)}^{0}(x, v)$, has no preferred v-direction, which entails that $\int \frac{d \Omega}{4 \pi} \xi_{(0)}^{0}(x, v)=0$ in the leading logarithmic approximation. Thus, the mean current conservation is not affected by the noise term.

\section{G. Mean Field Equations and Non-Abelian Ohm's law}

We have managed to obtain the following set of mean field equations, after integratingout the statistical fluctuations (from now on, we drop the bar to denote the mean fields)

$$
\begin{aligned}
v^{\mu} D_{\mu} \mathcal{J}^{\rho}(x, v) & =-m_{D}^{2} v^{\rho} v^{\mu} F_{\mu 0}(x)-\gamma v^{\rho} \int \frac{d \Omega_{\mathbf{v}^{\prime}}}{4 \pi} \mathcal{I}\left(\mathbf{v}, \mathbf{v}^{\prime}\right) \mathcal{J}^{0}\left(x, v^{\prime}\right)+\zeta^{\rho}(x, v), \\
D_{\mu} F^{\mu \nu} & =\int \frac{d \Omega_{\mathbf{v}}}{4 \pi} \mathcal{J}^{\nu}(x, v) .
\end{aligned}
$$

Here, we denote by $\zeta^{\rho}(x, v)$ the stochastic noise term identified in the preceding section, its correlator given by (7.48). We also introduced

$$
\gamma=\frac{g^{2}}{4 \pi} N T \ln (1 / g)
$$

which will be identified as (twice) the damping rate for the ultra-soft currents. We shall refer to (7.51a) as a Boltzmann-Langevin equation, as it accounts for particle interactions via a collision integral as well as for the stochastic character of the underlying fluctuations. 
The Boltzmann-Langevin equation (7.51a) has three distinct scale parameters, the temperature $T$, the Debye mass $m_{D}$, and the damping term $\gamma$. In the leading logarithmic approximation, these scales are well separated, $\gamma \ll m_{D} \ll T$. This is why (7.51) is dominated by different terms, depending on the momentum range considered. For hard momenta, (7.51a) is only dominated by the l.h.s., reducing it to the (trivial) current of hard particles moving on world lines. For momenta about the Debye mass, the term proportional to $m_{D}^{2}$ becomes equally important, while the noise term and the collision integral remain suppressed by $\gamma / m_{D}$. The resulting current is then given by (7.9), the HTL current. Momentum modes below the Debye mass are affected by the damping term of the collision integral. Close to the scale of the Debye mass, the higher order corrections to (7.9) are obtained as an expansion in $\gamma / v \cdot D$,

$$
\mathcal{J}^{\mu}(x, v)=\sum_{n=0}^{\infty} \mathcal{J}_{(n)}^{\mu}(x, v),
$$

where the current densities $\mathcal{J}_{(n)}^{\mu}(x, v)$ obey the differential equations

$$
\begin{aligned}
& v^{\mu} D_{\mu} \mathcal{J}_{(0)}^{\nu}(x, v)=-m_{D}^{2} v^{\nu} v^{\mu} F_{\mu 0}(x)+\zeta^{\nu}(x, v) \\
& v^{\mu} D_{\mu} \mathcal{J}_{(n)}^{\nu}(x, v)=-\gamma v^{\nu} \int \frac{d \Omega_{\mathbf{v}^{\prime}}}{4 \pi} \mathcal{I}\left(\mathbf{v}, \mathbf{v}^{\prime}\right) \mathcal{J}_{(n-1)}^{0}\left(x, v^{\prime}\right)
\end{aligned}
$$

Apart from the noise term, the leading order term in this expansion, $\mathcal{J}_{(0)}^{\nu}(x, v)$, coincides with the HTL current (7.9). All higher order terms $\mathcal{J}_{(n)}^{\mu}(x, v)$ are smaller by powers of $\sim(\gamma / v \cdot D)^{n}$, and recursively given by

$$
\begin{aligned}
& \mathcal{J}_{(0)}^{\nu}(x, v)=\int_{0}^{\infty} d \tau U(x, x-v \tau)\left\{-m_{D}^{2} v^{\nu} v^{\mu} F_{\mu 0}(x-v \tau)+\zeta^{\nu}(x-v \tau, v)\right\}, \\
& \mathcal{J}_{(n)}^{\nu}(x, v)=-\gamma \int_{0}^{\infty} d \tau U(x, x-v \tau) v^{\nu} \int \frac{d \Omega_{\mathbf{v}^{\prime}}}{4 \pi} \mathcal{I}\left(\mathbf{v}, \mathbf{v}^{\prime}\right) \mathcal{J}_{(n-1)}^{0}\left(x, v^{\prime}\right)
\end{aligned}
$$

This expansion is consistent with covariant current conservation. For every partial sum up to order $n$, we have

$$
D_{\mu}\left(J_{(0)}^{\mu}+J_{(1)}^{\mu}+\ldots+J_{(n)}^{\mu}\right)=0
$$

This expansion describes correctly how the presence of the collision integral modifies the ultra-soft current. However, it has a limited domain of validity. In particular, the overdamped regime where $v \cdot D \ll \gamma$ can not be reached, because the effective expansion parameter diverges.

Alternatively, one can separate the local from the non-local part of the collision integral to perform an expansion in the latter only. The effective expansion parameter is then $\gamma /(v \cdot D+\gamma)$, which has a better IR behaviour. We expect this expansion to be much better for the spatial than for the temporal component of $\mathcal{J}^{\rho}(x, v)$. This is so, because the term proportional to the non-local part $\mathcal{K}\left(\mathbf{v}, \mathbf{v}^{\prime}\right)$ of the collision integral in (7.51a) gives no contribution to the dynamical equations of the spatial component $J^{i}$, after angle averaging (7.51a) over the directions of $\mathbf{v}$. However, for the dynamical equation of the temporal 
component, this term precisely cancels the local damping term, which is of course a direct consequence of current conservation.

In this light, we decompose the current as in $(7.53)$ to find the differential equations

$$
\begin{aligned}
& \left(v^{\mu} D_{\mu}+\gamma\right) \mathcal{J}_{(0)}^{\rho}(x, v)=-m_{D}^{2} v^{\rho} v^{\mu} F_{\mu 0}(x)+\zeta^{\rho}(x, v) \\
& \left(v^{\mu} D_{\mu}+\gamma\right) \mathcal{J}_{(n)}^{\rho}(x, v)=\gamma v^{\rho} \int \frac{d \Omega_{\mathbf{v}^{\prime}}}{4 \pi} \mathcal{K}\left(\mathbf{v}, \mathbf{v}^{\prime}\right) \mathcal{J}_{(n-1)}^{0}\left(x, v^{\prime}\right)
\end{aligned}
$$

The retarded Green's function of the differential operator

$$
i\left(v^{\mu} D_{\mu}+\gamma\right) G_{\mathrm{ret}}(x, y ; v)=\delta^{(4)}(x-y)
$$

reads, for $t=x_{0}-y_{0}$,

$$
G_{\mathrm{ret}}(x, y ; v)_{a b}=-i \theta(t) \delta^{(3)}(\mathbf{x}-\mathbf{y}-\mathbf{v} t) \exp (-\gamma t) U_{a b}(x, y)
$$

and the iterative solution to the Boltzmann-Langevin equation obtains as

$$
\begin{aligned}
& \mathcal{J}_{(0)}^{\rho}(x, v)=\int_{0}^{\infty} d \tau \exp (-\gamma \tau) U(x, x-v \tau)\left\{-m_{D}^{2} v^{\rho} v^{j} F_{j 0}(x-v \tau)+\zeta^{\rho}(x-v \tau, v)\right\} \\
& \mathcal{J}_{(n)}^{\rho}(x, v)=\gamma \int_{0}^{\infty} d \tau \exp (-\gamma \tau) U(x, x-v \tau) v^{\rho} \int \frac{d \Omega_{\mathbf{v}^{\prime}}}{4 \pi} \mathcal{K}\left(\mathbf{v}, \mathbf{v}^{\prime}\right) \mathcal{J}_{(n-1)}^{0}\left(x-v \tau, v^{\prime}\right) .
\end{aligned}
$$

This expansion in consistent with current conservation, if the angle average of $\mathcal{J}_{(n)}^{0}$ vanishes for some $n$. This follows from taking the temporal component of (7.57) and averaging the equation over $\mathbf{v}$, to find

$$
\begin{aligned}
& D_{0} J_{(0)}^{0}+D_{i} J_{(0)}^{i}=-\gamma J_{(0)}^{0}, \\
& D_{0} J_{(n)}^{0}+D_{i} J_{(n)}^{i}=\gamma J_{(n-1)}^{0}-\gamma J_{(n)}^{0},
\end{aligned}
$$

for the individual contributions, and

$$
D_{\mu}\left(J_{(0)}^{\mu}+J_{(1)}^{\mu}+\ldots+J_{(n)}^{\mu}\right)+\gamma J_{(n)}^{0}=0
$$

for their sum, which is consistent if $\gamma J_{(n)}^{0}$ is vanishing for some $n$.

To leading order, the ultra-soft colour current $\mathcal{J}_{(0)}^{i}(x, v)$ in (7.60a) has the same functional dependence on the field strength and on the parallel transporter as the soft colour current (7.9). There is, however, an additional damping factor $e^{-\gamma \tau}$ in the integrand.

We shall now consider the overdamped regime (or quasi-local limit) of the above equations. Consider the mean field currents (7.60). The terms contributing to these currents are exponentially suppressed for times $\tau$ much larger than the characteristic time scale $1 / \gamma$. On the other hand, the fields occurring in the integrand vary typically very slowly, that is on time scales $\ll 1 / m_{\mathrm{D}}$. Thus, the quasi-local limit consists in the approximation

\footnotetext{
${ }^{\dagger \dagger}$ We thank D. Bödeker for pointing out a mistake in the reasoning presented in an earlier version.
} 


$$
U_{a b}(x, x-v \tau) \approx U_{a b}(x, x)=\delta_{a b}, \quad F_{j 0}(x-v \tau) \approx F_{j 0}(x)
$$

In this case the remaining integration can be performed. The solution for the spatial current $J^{i}(x)$ stems entirely from the leading order term (7.60a). All higher order corrections vanish, because they are proportional to

$$
\int \frac{d \Omega_{\mathbf{v}}}{4 \pi} \mathbf{v} \mathcal{K}\left(\mathbf{v}, \mathbf{v}^{\prime}\right)=0
$$

Therefore, we obtain for the spatial current from $(7.60)$

$$
J_{a}^{i}=\sigma E_{a}^{i}+\nu_{a}^{i}, \quad \sigma=\frac{4 \pi m_{D}^{2}}{3 N g^{2} T \ln (1 / g)}
$$

where $\sigma$ denotes the colour conductivity of the plasma. The noise term becomes

$$
\nu(x)=\frac{1}{\gamma} \int \frac{d \Omega_{\mathbf{v}}}{4 \pi} \zeta(x, v), \quad\left\langle\nu_{a}^{i}(x) \nu_{b}^{j}(y)\right\rangle=2 T \sigma \delta^{i j} \delta_{a b} \delta^{(4)}(x-y)
$$

The noise term appearing in the Yang-Mills equation becomes white noise within this last approximation. The fluctuation-dissipation theorem is fulfilled because the strength of the noise-noise correlator (7.64b) is precisely given by the dissipative term of (7.64a). This is the simplest form of the FDT. The colour conductivity in the quasi-local limit has been discussed by several authors in the literature [13,16].

For the complete set of gauge field equations in the quasi-local limit we still need to know $J^{0}(x)$. The iterative solution (7.60) gives $J_{(n)}^{0}(x)=0$ to any finite order. Therefore, we use instead the un-approximated dynamical equation for $\mathcal{J}^{0}(x, v)$, which yields, averaged over the directions of $\mathbf{v}$, current conservation. In combination with the solution (7.64a) for the spatial current, the Boltzmann-Langevin equation (7.51) finally becomes

$$
\begin{aligned}
D_{\mu} F^{\mu i} & =\sigma E^{i}+\nu^{i}, \\
D_{i} E^{i} & =J^{0} \\
D_{0} J^{0} & =-\sigma J^{0}-D_{i} \nu^{i} .
\end{aligned}
$$

It is worth pointing out that already in the leading logarithmic approximation the noise term appearing in the Yang Mills equation is not white, except in the local limit (7.64b). The noise in the Boltzmann-Langevin equation, on the other hand, is white (see $(\overline{7.49})$, when averaged over the directions of $\mathbf{v}$.

For numerical computations, which can in principle take into account the non-localities of the problem, it might be more convenient to work with the two set of equations (7.51), rather than with a non-local stochastic gauge field equation.

\section{THE QUANTUM PLASMA CLOSE TO EQUILIBRIUM}




\section{A. The quantum plasma from transport theory}

Up to now we have made an entirely classical derivation of a Boltzmann equation with collision integrals and stochastic sources, and we have finally derived the mean gauge field equations. The basic ingredients for such a derivation were the classical equations of motion and the classical statistical averages introduced in sect. III. The following natural step is quantizing the whole procedure in order to obtain quantum Boltzmann equations and the corresponding mean gauge field equations.

In order to quantize this formulation one has to abandon the concept of classical trajectories, and introduce commutators for all the canonical conjugate pairs of variables. One should also take into account that quantum particles are indistinguishable. The natural formulation of the quantum problem is then given in terms of Wigner functions and density matrices. We will not present a rigorous discussion of the quantum counterpart of our formulation, but rather present a minimal set of changes in our equations which allow to consider also quantum plasmas close to equilibrium. We leave for a future project a much more rigorous discussion based on first principles of the quantum formulation of the problem.

Our starting observation is that even in quantum plasmas the physics occurring at soft and ultra-soft scales can be encoded into classical or semiclassical equations. The reason for this is that the occupation number for soft modes close to equilibrium is very large, suggesting that a description in terms of classical equations might also be valid to describe the physics of the soft and ultra-soft scales in the quantum plasmas.

Therefore, in order to consider a quantum plasma, we shall need to make several changes. The first step consists in expanding the mean distribution function around the appropriate quantum statistical distribution function. For a plasma close to equilibrium, these are given by (6.6). We also change the normalisation of the distribution functions as indicated in sect. $\nabla 1$.

This suffices to obtain the correct quantum value for the Debye mass, appearing in the Vlasov equation, and thus to reproduce fully the HTL effective theory in the leading order in $g$ [6].

When fluctuations are considered as well, it is equally important to modify the classical correlator (7.25) to the corresponding quantum statistical one. For bosons, and for every internal degree of freedom, one has

$$
\begin{aligned}
\left\langle\delta f_{\mathbf{x}, p, Q} \delta f_{\mathbf{x}^{\prime}, p^{\prime}, Q^{\prime}}\right\rangle= & (2 \pi)^{3} \delta^{(3)}\left(\mathbf{x}-\mathbf{x}^{\prime}\right) \delta^{(3)}\left(\mathbf{p}-\mathbf{p}^{\prime}\right) \delta\left(Q-Q^{\prime}\right) \bar{f}_{\mathrm{B}}\left(1+\bar{f}_{\mathrm{B}}\right) \\
& +\tilde{g}_{2}^{\mathrm{B}}\left(\mathbf{x}, p, Q ; \mathbf{x}^{\prime}, p^{\prime}, Q^{\prime}\right)
\end{aligned}
$$

while the corresponding correlator for fermions is

$$
\begin{aligned}
\left\langle\delta f_{\mathbf{x}, p, Q} \delta f_{\mathbf{x}^{\prime}, p^{\prime}, Q^{\prime}}\right\rangle= & (2 \pi)^{3} \delta^{(3)}\left(\mathbf{x}-\mathbf{x}^{\prime}\right) \delta^{(3)}\left(\mathbf{p}-\mathbf{p}^{\prime}\right) \delta\left(Q-Q^{\prime}\right) \bar{f}_{\mathrm{F}}\left(1-\bar{f}_{\mathrm{F}}\right) \\
& +\tilde{g}_{2}^{\mathrm{F}}\left(\mathbf{x}, p, Q ; \mathbf{x}^{\prime}, p^{\prime}, Q^{\prime}\right) .
\end{aligned}
$$

The functions $\tilde{g}_{2}^{\mathrm{B} / \mathrm{F}}$ are related to the bosonic/fermionic two-particle correlation function. The above relations should be derived from first principles in a similar way as our equation (7.25). In the limit $\bar{f}_{\mathrm{B} / \mathrm{F}} \ll 1$ they reduce to the correct classical value. It has also to 
be pointed out that the correlators $(8.1)$ and (8.2) have been derived for the case of both an ideal gas of bosons and ideal gas of fermions close to equilibrium, matching the change described above. This can be taken as the correct answer in the case that the non-Abelian interactions can be treated perturbatively.

With the above in mind, we can now describe the minimal set of changes to our computations of sect. VII which allows to treat the quark-gluon plasma close to equilibrium. We will consider gluons in the adjoint representation with $C_{2}=N$, and $N_{F}$ quarks and $N_{F}$ antiquarks in the fundamental representation, with $C_{2}=1 / 2$. All particles carry two helicities. We will neglect the masses of the quarks, $m \ll T$.

The value of the the quantum Debye mass squared becomes

$$
m_{D}^{2}=-\frac{g^{2}}{\pi^{2}} \int_{0}^{\infty} d p p^{2}\left(N \frac{d \bar{f}_{\mathrm{B}}^{\mathrm{eq}}}{d p}+N_{F} \frac{d \bar{f}_{\mathrm{F}}^{\mathrm{eq}}}{d p}\right) .
$$

Evaluating explicitly the integral, one finds $m_{D}^{2}=g^{2} T^{2}\left(2 N+N_{F}\right) / 6$.

The correlator of colour currents densities are then modified, according to the changes mentioned above. Since we now consider different species of particles in (7.28) $C_{2} B_{C}$ is replaced by a sum over different species of particles. For the quark gluon plasma, all our equations of sect. VII remain valid if we replace $C_{2} B_{C}$ by

$$
\sum_{\text {species }} C_{2} B_{C}=\frac{2 N}{\pi} \int_{0}^{\infty} d p p^{2} \bar{f}_{\mathrm{B}}^{\mathrm{eq}}\left(1+\bar{f}_{\mathrm{B}}^{\mathrm{eq}}\right)+\frac{2 N_{F}}{\pi} \int_{0}^{\infty} d p p^{2} \bar{f}_{\mathrm{F}}^{\mathrm{eq}}\left(1-\bar{f}_{\mathrm{F}}^{\mathrm{eq}}\right) .
$$

It is curious that the relation (7.36) for the quantum values of the above quantities remains unchanged, that is,

$$
2 g^{2} \sum_{\text {species }} C_{2} B_{C}=4 \pi T m_{D}^{2}
$$

holds true also in the quark-gluon plasma. Since this combination appears in front of all our collision integrals, we find a universal value for the coefficient of (7.42), $\gamma$ for both the classical and quantum plasmas. This is always the case in the leading logarithmic approximation, if the IR cutoff used is of order $g m_{D}$, where $m_{D}$ would correspond to the classical or quantum Debye mass, respectively. The value $\gamma / 2$ can be identified with the damping rate of a hard transverse gluon [17.

With these observations, one does not need to repeat the computations that we performed in sect. VII. In particular, the final mean field equations of sect. VIIG only change in the value of the Debye mass.

\section{B. Comparison to related work}

Let us briefly comment on some related work. A similar philosophy to ours has already been followed by Selikhov [8]. He used the semiclassical limit of quantum transport equations for the Wigner functions associated to gluons and quarks, which reduce to our starting classical transport equations. He used a procedure of splitting both the Wigner functions 
and vector gauge fields into mean values and statistical fluctuations. A key point is how the statistical correlator of fluctuations in a quantum framework can be derived. Selikhov relied on the same type of statistical correlator as derived in (7.28). However, it should be stressed that this statistical correlator is only correct in the pure classical framework, for classical statistics. This can not reproduce the correct prefactors of the quantum collision integral. Also, the FDT is not satisfied in this case. Instead, the correct correlators are given by (8.1) and (8.2). Also, the colour current he found is not covariantly conserved. This is so because the non-local term in the collision integral (7.42), proportional to $\mathcal{K}\left(\mathbf{v}, \mathbf{v}^{\prime}\right)$, has been neglected.

The first to derive the mean field equations (7.51) and the related noise correlator (7.48) for the quantum plasma was Bödeker [12]. His approach uses the local version of the HTL effective action as the starting point to integrate-out the modes with momenta about the Debye mass. He profits from the observation that the soft field modes behave classically. This allows the definition of a classical thermal average with a weight given by the HTL Hamiltonian. Although the starting point and the techniques involved appear to be quite different from our approach, it remains intriguing that the integrating-out of momentum modes within a field theory results in the same effective theory as integrating-out statistical fluctuations within the present transport theory approach.

A different line has been followed by Arnold, Son and Yaffe [13], who realized that Bödeker's effective theory has a physical interpretation in terms of kinetic equations. They managed to derive the relevant collision term of the Boltzmann equation on phenomenological grounds.

Very recently, the quantum collision integral has been obtained within a quantum field theoretical setting by Blaizot and Iancu [14]. The derivation relies on a gauge covariant derivative expansion of the quantum field equations. While conceptually very different to our approach [1], the approximations used in [14] are very similar to the ones we performed. This is maybe not too surprising after all, as the explicit computations in both approaches are based on a consistent expansion in powers of the gauge coupling constant. It seems only that the concept of statistical fluctuations has not been introduced in [14], which may be a reason for why the source of stochastic noise, necessary for a correct macroscopic description of the plasma, has not yet been identified. The stochastic noise can probably be derived by considering the effects of higher order correlation functions [18].

Based on our approach [1], the quantum collision integral for the transport equation has been obtained as well by Valle [19]. He started from the HTL effective theory and found the correct damping rate after imposing a fluctuation-dissipation relation. The noise term in his final equations is however missing, which again would entail that the system abandons equilibrium.

Finally, it is interesting that the collision integral can be interpreted in terms of Feynman diagrams [12,14]. Bödeker also made a diagrammatic derivation of his effective theory [12]. This is a much more lengthy and cumbersome task, and shows, on the other hand, the very efficiency of a kinetic approach, as it corresponds to a re-organisation of the perturbative series. 


\section{DISCUSSION}

We have presented a self-consistent approach to study classical non-Abelian plasmas. Let us summarize here again our starting assumptions to derive the effective transport equations. A system of point particles carrying non-Abelian charges is considered. Their microscopic equations of motion are the Wong equations. In order to describe an ensemble of these particles, we introduced an ensemble average, which takes also the colour charges as dynamical variables into account. This yields finally a set of transport equations for both mean quantities and statistical fluctuations, and gives a recipe to obtain explicitly the collision integrals for macroscopic transport equations. This approach is consistent with gauge invariance, and admits systematic approximations. Most particularly, it does not rely on close-to-equilibrium situations. These techniques, applied since long to Abelian plasmas, have never been fully exploited for the non-Abelian case. Our approach is aimed at closing this gap in the literature of non-Abelian plasmas.

We applied this method to non-Abelian plasmas close to thermal equilibrium. A sufficiently small gauge coupling parameter is at the basis for a systematic expansion of the dynamical equations. Neglecting fluctuations yields to leading order the known non-local expression for the soft current in terms of the soft gauge fields (HTL approximation). Integrating-out, in addition, the fluctuations to leading logarithmic order, that is with momenta about $m_{D}$, results in a Boltzmann-Langevin equation for the ultra-soft modes,

$$
v^{\mu} D_{\mu} \mathcal{J}^{\rho}(x, v)=-m_{D}^{2} v^{\rho} v^{\mu} F_{\mu 0}(x)-\gamma v^{\rho} \int \frac{d \Omega_{\mathbf{v}^{\prime}}}{4 \pi} \mathcal{I}\left(\mathbf{v}, \mathbf{v}^{\prime}\right) \mathcal{J}^{0}\left(x, v^{\prime}\right)+\zeta^{\rho}(x, v)
$$

It contains a collision term and a related noise term, with $\gamma=g^{2} N T \ln (1 / g) / 4 \pi$, while the stochastic source $\zeta$ obeys

$$
\left\langle\zeta_{a}^{\mu}(x, v) \zeta_{b}^{\nu}\left(y, v^{\prime}\right)\right\rangle=2 \gamma T m_{D}^{2} v^{\mu} v^{\prime \nu} \mathcal{I}\left(\mathbf{v}, \mathbf{v}^{\prime}\right) \delta_{a b} \delta^{(4)}(x-y) .
$$

The above is completed with the Yang-Mills equations. Solving the Boltzmann-Langevin equation (see sect. VIIG), one obtains the Yang-Mills equation for the ultra-soft fields

$$
\left[D_{\mu}, F^{\mu \nu}\right](x)=\int \frac{d \Omega_{\mathbf{v}}}{4 \pi} \mathcal{J}^{\nu}(x, v)
$$

Surprisingly, the dynamical equations are the same for both classical and quantum plasmas, the only difference being the value for the Debye mass. This conclusion relies also on the use of an infrared cut-off of order $g m_{D}$, where $m_{D}$ is the classical or quantum Debye mass. For the quantum plasmas, our result agrees with the quantum collision integrals found in the literature using different methods [12 [14]. The main effect of the fluctuations with momenta about the Debye mass is the introduction of a damping term and a source of stochastic noise into the above expression. Note also that the damping coefficient $\gamma$ is the same for classical and quantum plasmas.

Our work establishes a link even beyond the one-loop level between the classical transport theory approach as presented here, and a full quantum field theoretical treatment. It would

be most desirable if this connection could be further substantiated. This should also yield 
a quantitative criterion for the applicability of the -technically speaking- much simpler approach based on the classical point particle picture.

Let us finally emphasize that the same IR problems, which are due to the unscreened magnetic modes, appear both for classical and quantum plasmas. This suggests that the solution for these IR divergences might also be the same in the two cases. Therefore, it seems profitable to seek for a solution to this problem in the much simpler framework of classical transport theory, rather than in a quantum field theoretical approach.

\section{ACKNOWLEDGMENTS}

We wish to thank Dietrich Bödeker for discussions.

\section{APPENDIX A: DARBOUX VARIABLES}

The statistical averages defined in sect. III have to be performed in phase space. The colour charges $Q_{a}$ are not real phase space variables [6]. It is possible to define the set of Darboux variables associated to the $Q_{a}$ charges.

For $S U(2)$ we define the new set of variables $\phi, \pi, J$ by the transformation [6]

$$
Q_{1}=\cos \phi \sqrt{J^{2}-\pi^{2}} \quad Q_{2}=\sin \phi \sqrt{J^{2}-\pi^{2}} \quad Q_{3}=\pi
$$

where $\pi$ is bounded by $-J \leq \pi \leq J$. The variables $\phi, \pi$ form a canonically conjugate pair, while $J$ is fixed by the value of the quadratic Casimir, which is constant under the dynamical evolution. One can define Poisson brackets with these canonical variables, under which the colour charges form a representation of $S U(2),\left\{Q_{a}, Q_{b}\right\}_{P B}=f_{a b c} Q_{c}$. With the above change of variables, one can easily fix the value of the representation normalization constant $c_{R}$ introduced in (2.9). From the condition $\int d Q=1$ one finds $c_{R}=1 / 2 \pi \sqrt{q_{2}}$. From the condition $\int d Q Q_{a} Q_{b}=C_{2} \delta_{a b}$ one gets $q_{2}=3 C_{2}$. This entirely fixes the value of $c_{R}$ as a function of $C_{2}$.

The Darboux variables associated to $S U(3)$ were defined in [6], and will not be discussed explicitly here.

We should also comment that in the pure classical framework, $C_{2}$ carries dimensions of $\hbar c$. After quantization, the quadratic Casimirs should take quantized values proportional to $\hbar$. The Poisson brackets then have to be replaced by commutators.

\section{APPENDIX B: CONSISTENT CURRENT CONSERVATION}

In this appendix we verify explicitly the identity

$$
0=\left[\bar{D}_{\mu}, J_{\text {fluc }}^{\mu}\right]+g\left[a_{\mu}, \delta J^{\mu}\right]+g\left[a_{\mu},\left\langle J_{\text {fluc }}^{\mu}\right\rangle\right],
$$

which is at the basis for the proof of the consistent current conservation of both the mean field and the fluctuation current in sect. $\mathrm{VB}$. The following check is algebraic, and it 
will make use of symmetry arguments like the antisymmetry of the commutator and the tensors $\bar{F}_{\mu \nu}, f_{\mu \nu}$, and of the cyclic identity $\left[t_{a},\left[t_{b}, t_{c}\right]\right]+\left[t_{b},\left[t_{c}, t_{a}\right]\right]+\left[t_{c},\left[t_{a}, t_{b}\right]\right]=0$. The identity $\left[\bar{D}_{\mu}, \bar{D}_{\nu}\right]=g \bar{F}_{\mu \nu}$ is employed as well. To simplify the computation, we will seperate the fluctuation part of the field strength (4.2c) into the term linear and quadratic in $a$, according to

$$
f_{\mu \nu}=f_{1, \mu \nu}+f_{2, \mu \nu}, \quad f_{1, \mu \nu}=\left[\bar{D}_{\mu}, a_{\nu}\right]-\left[\bar{D}_{\nu}, a_{\mu}\right], \quad f_{2, \mu \nu}=g\left[a_{\mu}, a_{\nu}\right] .
$$

Recall furthermore, using (5.7c) and (5.8b), that

$$
\begin{aligned}
& J_{\text {fluc }}^{\mu}=\left[\bar{D}_{\nu}, f_{2}^{\nu \mu}\right]+g\left[a_{\nu}, f_{1}^{\nu \mu}+f_{2}^{\nu \mu}\right] \\
& \delta J^{\mu}=\left[\bar{D}_{\nu}, f_{1}^{\nu \mu}\right]+g\left[a_{\nu}, \bar{F}^{\nu \mu}\right]+J_{\text {fluc }}^{\mu}-\left\langle J_{\text {fluc }}^{\mu}\right\rangle
\end{aligned}
$$

are functions of the fluctuation field $a$. The first term of (B1) reads, after inserting $J_{\text {fluc }}$ from (B3),

$$
\left[\bar{D}_{\mu}, J_{\text {fluc }}^{\mu}\right]=\left[\bar{D}_{\nu},\left[\bar{D}_{\mu}, f_{2}^{\mu \nu}\right]\right]+g\left[\bar{D}_{\nu},\left[a_{\mu}, f_{1}^{\mu \nu}\right]\right]+g\left[\bar{D}_{\nu},\left[a_{\mu}, f_{2}^{\mu \nu}\right]\right]
$$

Using $\delta J$ from (B4), it follows for the second term of (B1)

$$
g\left[a_{\mu}, \delta J^{\mu}\right]=g^{2}\left[a_{\nu},\left[a_{\mu}, \bar{F}^{\mu \nu}\right]\right]+g\left[a_{\nu},\left[\bar{D}_{\mu}, f_{1}^{\mu \nu}\right]\right]+g\left[a_{\nu}, J_{\text {fluc }}^{\nu}\right]-g\left[a_{\nu},\left\langle J_{\text {fluc }}^{\nu}\right\rangle\right]
$$

The last term of $(\mathbb{B} 6)$ will be canceled by the last term in $(\mathbb{B} 1)$. We show now that the first three terms of $(\overline{B 5})$ and $(\mathrm{B} 6)$ do cancel one by one. The first term in (B5) can be re-written as

$$
\left[\bar{D}_{\nu},\left[\bar{D}_{\mu}, f_{2}^{\mu \nu}\right]\right]=\left[\left[\bar{D}_{\nu}, \bar{D}_{\mu}\right], f_{2}^{\mu \nu}\right]-\left[\bar{D}_{\nu},\left[\bar{D}_{\mu}, f_{2}^{\mu \nu}\right]\right]=\frac{1}{2} g\left[\bar{F}_{\nu \mu}, f_{2}^{\mu \nu}\right]
$$

Similarly, the first term of (B6) yields

$$
g^{2}\left[a_{\nu},\left[a_{\mu}, \bar{F}^{\mu \nu}\right]\right]=-g^{2}\left[\bar{F}^{\mu \nu},\left[a_{\nu}, a_{\mu}\right]\right]-g^{2}\left[a_{\nu},\left[a_{\mu}, \bar{F}^{\mu \nu}\right]\right]=-\frac{1}{2} g\left[\bar{F}_{\mu \nu}, f_{2}^{\nu \mu}\right] .
$$

For the second term in (B5) we have

$$
g\left[\bar{D}_{\nu},\left[a_{\mu}, f_{1}^{\mu \nu}\right]\right]=g\left[a_{\mu},\left[\bar{D}_{\nu}, f_{1}^{\mu \nu}\right]\right]+g\left[\left[\bar{D}_{\nu}, a_{\mu}\right], f_{1}^{\mu \nu}\right]=-g\left[a_{\mu},\left[\bar{D}_{\nu}, f_{1}^{\nu \mu}\right]\right]
$$

which equals (minus) the second term of (B6). Finally, consider the third term of (B6),

$$
\begin{aligned}
g\left[a_{\nu}, J_{\text {fluc }}^{\nu}\right] & =g^{2}\left[a_{\nu},\left[\bar{D}_{\mu},\left[a^{\mu}, a^{\nu}\right]\right]\right]+g^{2}\left[a_{\nu},\left[a_{\mu}, f^{\mu \nu}\right]\right] \\
& =\frac{1}{2} g\left[f_{2}^{\mu \nu}, f_{1, \mu \nu}\right]-g\left[\bar{D}_{\mu},\left[f_{2}^{\mu \nu}, a_{\nu}\right]\right]-\frac{1}{2} g\left[f_{2}^{\mu \nu}, f_{1, \mu \nu}\right] \\
& =-g\left[\bar{D}_{\mu},\left[a_{\nu}, f_{2}^{\nu \mu}\right]\right]
\end{aligned}
$$

which equals (minus) the third term of (B5). This establishes (B1). 
[1] D.F. Litim and C. Manuel, Phys. Rev. Lett. 82, 4981 (1999), hep-ph/9902430.

[2] D. J. Gross, R. D. Pisarski, and L. G. Yaffe, Rev. Mod. Phys. 53, 43 (1981).

[3] H.-Th. Elze and U. Heinz, Phys. Rep. 189, 81 (1989).

[4] R.D. Pisarski, Phys. Rev. Lett 63, 1129 (1989);

E. Braaten and R.D. Pisarski, Nucl. Phys. B337, 569 (1990);

J. Frenkel and J.C. Taylor, Nucl. Phys. B334, 199 (1990);

J. C. Taylor and S.M.H. Wong, Nucl. Phys. B346, 115 (1990);

R. Efraty and V.P. Nair, Phys. Rev. Lett. 68, 2891 (1992);

R. Jackiw and V.P. Nair, Phys. Rev. D48, 4991 (1993);

J.P. Blaizot and E. Iancu, Phys. Rev. Lett 70, 3376 (1993); Nucl. Phys. B417, 608 (1994).

[5] U. Heinz, Phys. Rev. Lett. 51, 351 (1983); Ann. Phys. 161, 48 (1985); ibid 168, 148 (1986).

[6] P.R. Kelly, Q. Liu, C. Lucchesi and C. Manuel, Phys. Rev. Lett 72, 3461 (1994); Phys. Rev. D 50, 4209 (1994).

[7] Yu. L. Klimontovich, "Statistical Physics", Harwood Academic Publishers, (1986);

E. Lifshitz and L. Pitaevskii, "Physical Kinetics", Pergamon Press, Oxford (1981).

[8] A.V. Selikhov, Phys. Lett. B268, 263 (1991), Erratum Phys. Lett. B285, 398 (1992).

[9] A.V. Selikhov and M. Gyulassy, Phys. Rev. C 49, 1726 (1994).

[10] Y.A. Markov and M.A. Markova, Theor. Math. Phys. 103, 444 (1995).

[11] S. Wong, Nuovo Cim. 65A, 689 (1970).

[12] D. Bödeker, Phys. Lett. B426, 351 (1998); preprint NBI-HE-99-04, hep-ph/9903478; preprint NBI-HE-99-13, hep-ph/9905239.

[13] P. Arnold, D. T. Son, and L. G. Yaffe, Phys. Rev. D 59; 105020 (1999); hep-ph/9901304.

[14] J. P. Blaizot and E. Iancu, preprint Saclay-T99/026, CERN-TH/99-71, hep-ph/9903389.

[15] L.F. Abbott, Nucl. Phys. B185, 189 (1981);

H.-Th. Elze, Z. Phys. C47, 647 (1990).

[16] A.V. Selikhov and M. Gyulassy, Phys. Lett. B316, 373 (1993);

H. Heiselberg, Phys. Rev. Lett. 72, 3013 (1994).

[17] R. D. Pisarski, Phys. Rev. D47, 5589 (1993).

[18] E. Iancu, private communication.

[19] M. A. Valle Basagoiti, preprint U. Pais Vasco EHU-FT/9905, hep-ph/9903462. 